\title{
Modeling Shear Strength of Medium- to Ultra-High- Strength Concrete Beams with Stirrups using SVR and Genetic Algorithm
}

Chun-song Jiang ( $\sim$ jchs2015@glut.edu.cn )

Guilin University of Technology https://orcid.org/0000-0001-6660-9933

Gui-Qin Liang

Guilin University of Technology

\section{Research Article}

Keywords: Shear strength, Ultra-high strength concrete beams, Stirrups, Support vector regression, Genetic algorithm

Posted Date: April 16th, 2021

DOl: https://doi.org/10.21203/rs.3.rs-338840/v1

License: (c) (i) This work is licensed under a Creative Commons Attribution 4.0 International License.

Read Full License

Version of Record: A version of this preprint was published at Soft Computing on July 12th, 2021. See the published version at https://doi.org/10.1007/s00500-021-06027-2. 


\title{
Modeling Shear Strength of Medium- to Ultra-High-Strength Concrete Beams with Stirrups using SVR and Genetic Algorithm
}

\author{
Chun-Song Jiang 1,2,* Gui-Qin Liang ${ }^{3}$
}

1. Guangxi Key Laboratory of New Energy and Building Energy Saving, Guilin 541004, Guangxi, China;

2. College of Civil and Architecture Engineering, Guilin University of Technology, Guilin 541004, Guangxi, China;

3. School of Information and Communication, Guilin University of Technology, Guilin 541004, Guangxi, China.

*Corresponding author at: College of Civil and Architecture Engineering, Guilin University of Technology, Guilin 541004, Guangxi, China.

E-mail address: jchs2015@glut.edu.cn (C.- S. Jiang).

\begin{abstract}
This paper presents a data-driven machine learning approach of support vector regression (SVR) with genetic algorithm (GA) optimization approach called SVR-GA for predicting the shear strength capacity of medium-to ultra-high strength concrete beams with longitudinal reinforcement and vertical stirrups. 148 experimental samples collected with different geometric, material and physical factors from literature were utilized for SVR-GA with 5-fold cross validation. Shear influence factors such as the stirrup spacing, the beam width, the shear span-to-depth ratio, the effective depth of the beam, the concrete compressive and tensile strength, the longitudinal reinforcement ratio, the product of stirrup ratio and stirrup yield strength were served as input variables. The simulation results show that the predicted shear strength of SVR-GA model can achieve high accuracy based on testing set with a coefficient of determination $\left(R^{2}\right)$ of 0.9642 , root mean squared error (RMSE) of 1.4685 and mean absolute error (MAE) of 1.0216 superior to that for traditional SVR model with $0.9379,2.0375$ and 1.4917 . The sensitivity analysis reveals that the most important variables affecting the prediction of the shear strength are shear span-to-depth ratio, concrete compressive strength, reinforcement ratio and the product of stirrup ratio and stirrup yield strength. Three-dimensional input/output maps can vividly reflect the nonlinear variation of the shear strength with the two coupling variables. All in all, the proposed SVR-GA model presents an effective and accurate artificial intelligence technology for modeling the shear strength of ultra-high strength concrete beams with stirrups.
\end{abstract}

Keywords: Shear strength; Ultra-high strength concrete beams; Stirrups; Support vector regression; Genetic algorithm

Received: 6 March 2021

\section{Introduction}

The shear failure of reinforced concrete beams with stirrups is a common concern of structural engineers (Collins et al. 2009; Sagaseta and Vollum 2011; Słowik 2014). However, it is 
difficult to predict the shear failure accurately due to the influence of a large number of parameters, such as the stirrup spacing, the width and effective depth of the beam, shear span-to-depth ratio, stirrup ratio, longitudinal reinforcement ratio, tensile compressive strength of concrete, and stirrup yield strength. This difficulty is particularly evident in ultra-high strength concrete (UHSC) and ultra-high performance concrete (UHPC) beams (Hossain et al. 2017).

In order to accurately estimate the shear capacity of the UHPC beam, the shear capacity is artificially divided into concrete shear capacity and stirrup yield shear capacity. An additional shear contribution of steel fiber would be added while adding steel fiber into concrete. Further, an additional shear contribution of the pin would be superimposed when taking the pin action of longitudinal reinforcement into account (Yoo and Yoon 2016; Marì Bernat et al. 2020). However, these factors are not independent of each other, and there is a coupling effect between them. For example, the residual tensile stress between cracks will be increased by appropriately adding the steel fiber while both increasing the shear contribution of the fiber and concrete.

A variety of normative formulas or models are proposed to solve problems in engineering applications. However, there are great differences in selecting main variables affecting the shear strength, such as the code of China for the design of concrete structures (GB 50010-2010 (Press 2010)) uses the concrete tensile strength to calculate the shear strength while the American concrete structure design code (ACI318-14 (ACI Committee 318 2014)) adopts the concrete compressive strength for that. The tensile strength and compressive strength of concrete are both considered by the Chinese highway and bridge code (JTG 3362-2018 (Ministry of Transport of China 2018)). Although there is little difference between UHPC and normal concrete in the ratio of tensile strength to compressive strength. The addition of steel fibers has greatly affected the tensile strength of UHPC while almost no influence on the compressive strength (Hassan et al. 2012; Krassowska et al. 2019). It is noteworthy that most of the formulas in the codes have been tested on the limited data which is just an extension of the existing empirical formulas for the shear strength of medium and high strength concrete beams, without fully considering and utilizing the ultra-high mechanical properties of ultra-high strength concrete.

Traditional models/equations with low accuracy mainly rely on basic expressions and step-by-step refinement process, it is urgent to proposed more accurate method to calculate the shear strength of UHPC beams. Recently, the data-driven machine learning methods have attracted extensive attention. Artificial neural network (Açikgenç et al. 2015; Golafshani et al. 2015; Hossain et al. 2017), adaptive fuzzy neural network (Mansouri et al. 2016; Nguyen et al. 2020), Gaussian process regression (Hoang et al. 2016; Guo and Hesthaven 2018) and support vector machine (Pal and Deswal 2011; Farfani et al. 2015) have been widely applied to model the mechanical properties of concrete structures.

Machine learning uses samples with input and output for training models that can be used to predict the output of new inputs. At present, machine learning has been used to predict the shear capacity of normal concrete beams or ultra-high strength concrete beams without stirrups (Solhmirzaei et al. 2020; Zhang et al. 2020). However, few studies were found for applying machine learning approach to predict the shear capacity of ultra-high strength concrete beams with stirrups. Support vector machine (SVM) is one of machine learning methods based on statistical learning theory of structural risk minimization. It is called support vector regression (SVR) for solving regression problems. SVR has the advantages of fast learning, global optimization, avoiding local minimization and excellent generalization ability (Çevik et al. 2015). The main 
problem of SVR is to choose proper parameters and kernel function, which needs to take time for trial and error (Yu et al. 2006; Ccoicca 2013). GA has been used as a powerful optimization tool to solve a variety of academic and engineering problems (Yan and Lin 2016; Chatterjee et al. 2017; Umeonyiagu and Nwobi-Okoye 2019). In order to select the optimal parameters quickly, genetic algorithm (GA) is introduced, which is an algorithm that simulates biological evolution process to search for global optimal solution.

In this study, a machine learning model combining genetic algorithm and support vector regression called hybrid SVR-GA model was proposed to predict the shear strength of ultra-high strength concrete beams with stirrups. Furthermore, the hybrid SVR-GA model was applied with 148 experimental results of medium- to ultra-high strength concrete beams with horizontal longitudinal reinforcement and vertical stirrups collected from previous studies. The 5-fold cross-validation method was adopted for model training and model validation. The optimal penalty coefficient and kernel parameters of SVR model were obtained by GA. And then the hybrid SVR-GA with optimal parameters was employed to predict the shear strength of ultra-high strength concrete beams with stirrups. It is expected that the shear strength of medium- to ultra-high strength concrete beams with any combination of design parameters can be predicted accurately providing guides for optimal design.

\section{Data Construction}

The experimental dataset in this study was collected from previously published works discussing about the shear strength of the simply supported beam (ZHANG HZ 2005; Magureanu et al. 2010; Ji et al. 2011; Baby et al. 2014; Xu et al. 2014; Kamal et al. 2014; Zhou JM and Chen S 2015; Hou et al. 2015; Jin et al. 2015; Lim and Hong 2016; Pansuk et al. 2017; Smarzewski 2018; Yousef et al. 2018; Mészöly and Randl 2018; Hasgul et al. 2019; Zheng et al. 2019; Krassowska et al. 2019; Qi et al. 2020; Wang et al. 2020). The typical RC beam with stirrups and its geometric parameters are illustrated in Fig. 1. The paper review helped determine the factors affecting the beam's shear capacity (Russo et al. 2004; Olalusi and Viljoen 2020). Various related factors used to predict ultimate shear capacity $(\mathrm{Vu})$ are shown in Table 1 , including different geometric, material and physical factors, such as stirrup spacing $(s)$, beam width $(b)$, shear span-to-depth ratio $(a / d)$, effective depth of the beam $(d)$, concrete compressive strength $\left(f_{\mathrm{c}}\right)$, concrete tensile strength $\left(f_{\mathrm{t}}\right)$, longitudinal reinforcement ratio $(\rho)$, as well as the product of the stirrup ratio and the stirrup yield strength $\left(\rho_{\mathrm{sv}} f_{\mathrm{yv}}\right)$. In this study, the normalized ultimate shear strength (i.e., $v_{\mathrm{u}}=V_{\mathrm{u}} /(b d)$ (Zhang et al. 2020)) was used as a measure for evaluating the shear resistance of the beam.

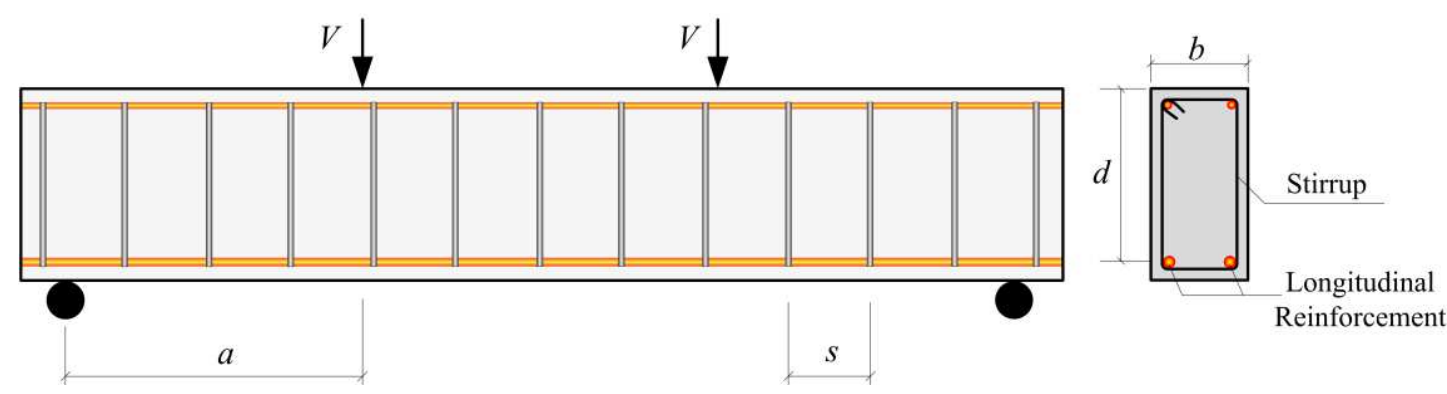

Fig. 1 Typical RC beam with stirrups and its geometric parameters 
Table 1 Initial statistical parameters for the experimental dataset

\begin{tabular}{llllllllll}
\hline Parameter & $s(\mathrm{~mm})$ & $b(\mathrm{~mm})$ & $a / d$ & $d(\mathrm{~mm})$ & $f_{\mathrm{c}}(\mathrm{MPa})$ & $f_{\mathrm{t}}(\mathrm{MPa})$ & $\rho(\%)$ & $\rho_{\mathrm{sv}} f_{\mathrm{yv}}(\mathrm{MPa})$ & $v_{\mathrm{u}}(\mathrm{MPa})$ \\
\hline Minimum & 60.00 & 40.00 & 0.79 & 114.00 & 23.60 & 1.96 & 0.50 & 12.02 & 1.17 \\
Mean & 179.10 & 122.47 & 2.67 & 277.39 & 109.60 & 7.49 & 3.78 & 274.29 & 12.11 \\
Maximum & 1000.00 & 200.00 & 5.00 & 430.00 & 232.10 & 24.18 & 13.57 & 1045.00 & 31.08 \\
Median & 151.00 & 120.00 & 2.50 & 270.27 & 116.70 & 6.14 & 3.25 & 141.74 & 9.67 \\
Std & 116.51 & 51.59 & 1.02 & 81.40 & 53.36 & 4.67 & 2.76 & 256.32 & 8.55 \\
Cov & 0.65 & 0.42 & 0.38 & 0.29 & 0.49 & 0.62 & 0.73 & 0.93 & 0.71 \\
\hline
\end{tabular}

*Std and Cov denotes the standard deviation and the coefficient of variable, respectively. When there was no test value of tensile strength $f_{\mathrm{t}}, 0.75$ times of splitting strength was taken (Zheng et al. 2019).

\section{Machine Learning Methods}

\subsection{Support vector regression (SVR)}

Suppose a sample is represented by $\left\{\left(\boldsymbol{x}_{\mathrm{i}}, y_{\mathrm{i}}\right), i=1,2, \ldots, \mathrm{n}\right\}$, where $\boldsymbol{x}_{\mathrm{i}}$ and $y_{\mathrm{i}}$ correspond to its input and output respectively. In SVR, the fundamental idea is to map $x_{\mathrm{i}}$ to a high- dimensional feature space $\boldsymbol{F}$ through a nonlinear mapping function $\Phi\left(x_{\mathrm{i}}\right)$. The goal of SVR is to perform linear regression in this space and find the following linear equation (Vapnik et al. 1997) defined in Eq. (1).

$$
f(x)=\boldsymbol{w} \cdot \Phi(x)+b, \Phi: \boldsymbol{R}^{n} \rightarrow \boldsymbol{F}, \boldsymbol{w} \in \boldsymbol{F}
$$

Where $w$ and $b$ are the normal vector and scalar, respectively. They can be derived from the minimized regularization risk function (Vapnik et al. 1997) showed in Eq. (2) as follows:

$$
\begin{aligned}
& \min R\left(\alpha, \alpha^{*}\right)=\sum_{i, j=1}^{n}\left(\alpha_{i}-\alpha_{i}^{*}\right)\left(\alpha_{j}-\alpha_{j}^{*}\right) K\left(x_{i}, x_{j}\right)+\sum_{i=1}^{n} \alpha_{i}\left(\varepsilon-y_{i}\right)+\sum_{i=1}^{n} \alpha_{i}\left(\varepsilon+y_{i}\right) \\
& \text { Subject to }\left\{\begin{array}{c}
\sum_{i=1}^{n}\left(\alpha_{i}-\alpha_{i}^{*}\right)=0 \\
\alpha_{i}, \alpha_{i}^{*} \in[0, C]
\end{array}\right.
\end{aligned}
$$

Where $C$ is the positive penalty coefficient, which is the tradeoff between model training error and model flatness. $\varepsilon$ is the insensitivity coefficient, which determines the error tolerance. $\alpha_{\mathrm{i}}$ and $\alpha_{\mathrm{i}}^{*}$ are the Lagrange multipliers related to the constraint. $K\left(x, x_{i}\right)=\Phi(x) \cdot \Phi\left(x_{i}\right)$ is the kernel function. After determining $\alpha_{i}, \alpha_{i}^{*}$ and $b$, the linear function in Eq. (1) can be expressed explicitly by Eq. (3).

$$
f(x)=\sum_{i=1}^{n}\left(\alpha_{i}-\alpha_{i}^{*}\right) K\left(x, x_{i}\right)+b
$$

One of the key problems in the application of SVR model is to select an appropriate kernel function. Four kinds of kernel functions are commonly used, namely linear, polynomial, sigmoid and Gaussian (radial basis) (Çevik et al. 2015). Considering the efficiency and reliability, especially in the face of a variety of parameters, Gaussian kernel function is selected here as shown below:

$$
K\left(x, x_{i}\right)=\exp \left(-\gamma\left\|x-x_{i}\right\|^{2}\right)
$$

Where $\gamma$ is the kernel parameter. The generalization ability of SVR depends on the proper setting of parameters $C, \gamma$ and $\varepsilon$. Genetic algorithm is used to determine the optimal value of parameters. 


\subsection{Genetic algorithm (GA)}

In the past few years, GA has been applied as an effective tool to solve different optimization problems in engineering and academia (Yan and Lin 2016; Chatterjee et al. 2017; Umeonyiagu and Nwobi-Okoye 2019). GA is not only a global optimization search algorithm by simulating the process of natural selection and biological evolution (Taylor 1994), but also a probabilistic parallel optimization method, which can adjust the search direction adaptively without the need of certain rules. The main characteristic is that it acts directly on the target object and uses the fitness function instead of the cost function without the requirement of function continuity and derivability. The complex problem can be solved by three genetic operations: selection, crossover and mutation.

In the GA, search parameters are binary encoded to produce many binary strings called chromosomes. Multiple chromosomes form an initial population. The aim is to get a qualified set of chromosomes after limited generations. For this purpose, a fitness function associated with chromosomes is defined. The higher fitness, the higher probability of chromosome selection, and then the selected chromosomes are crossed and mutated to produce a new population. Finally, a chromosome with the best fitness is obtained after finite generations, which is decoded to obtain optimal parameters. From this process, it can be seen that GA is independent of the specific domain of the problem and very robust to various optimization problems. The searching efficiency of GA is affected by some factors, such as population size, crossover probability and mutation probability, but it is out the scope of our paper

\subsection{The hybridized SVR-GA model}

The main problem of SVR model is to select appropriate kernel function and hyper parameters, which is time consuming work (Çevik et al. 2015). GA can be employed to quickly select the optimal parameters for SVR model. The flow diagram of proposed hybrid SVR-GA model was shown in Fig. 2. In the SVR-GA model, the population size was 50, while the evolutionary iteration was 200 generations, and the probability of crossover and mutation were 0.8 and 0.09 , respectively. The SVR parameters, $C$ and $\gamma$ were binary encoded while $\varepsilon$ was always 0.01 . For better evolution, the fitness function here adopts the correlation coefficient, which is calculated by the experimental value and predicted value of the SVR model. Higher fitness value means higher ranking for a chromosome, while a lower ranking chromosome is less likely to be selected. According to the principles of survival of the fittest, the latest generation chromosome with the best fitness can be decoded as the approximate optimal parameter after generation after generation of selection, crossover, and mutation. In the end, the optimal parameters can be obtained for the SVR model used for data training and model validation, and the well trained SVR-GA model for prediction with high accuracy can be obtained. 


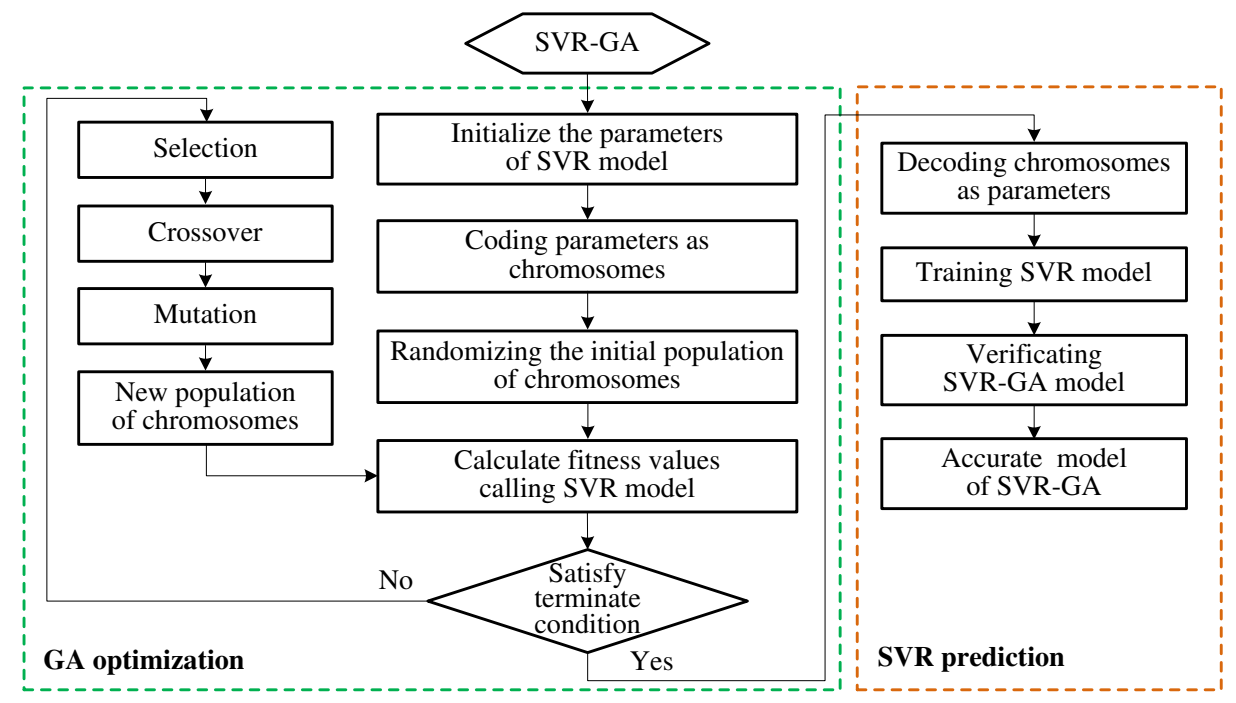

Fig. 2 Flow diagram of hybrid SVR-GA model

\section{Model implementation and performance metrics}

The dataset containing 148 experimental samples gathered from literature was split into training set and testing set for SVR-GA model. $16 \%$ of the dataset (24 samples) were randomly selected as testing set, which were not involved in the model training. The remaining $84 \%$ of the dataset (124 samples) were used to train and validate the model. To accurately evaluate the prediction ability of the model preventing from over-fitting and under-fitting conditions, a five-fold cross-validation method was utilized. It means the training set was divided into five equally sized subsets. Each fold was used to validate and obtain the optimal hyperparameters (i.e., $C, \gamma)$ of SVR model by GA, while the other four folds were used to train SVR model and get parameters $\boldsymbol{w}$ and $b$. In this process, one SVR-GA sub-model $\left(f_{i}\right)$ is generated for each fold. Over all folds, the average training error was calculated through five SVR-GA sub-model using all training set. The final prediction model of SVR-GA takes the average of the five sub-model predictions. More details for the five-fold cross-validation method in modeling process were shown in Fig. 3.

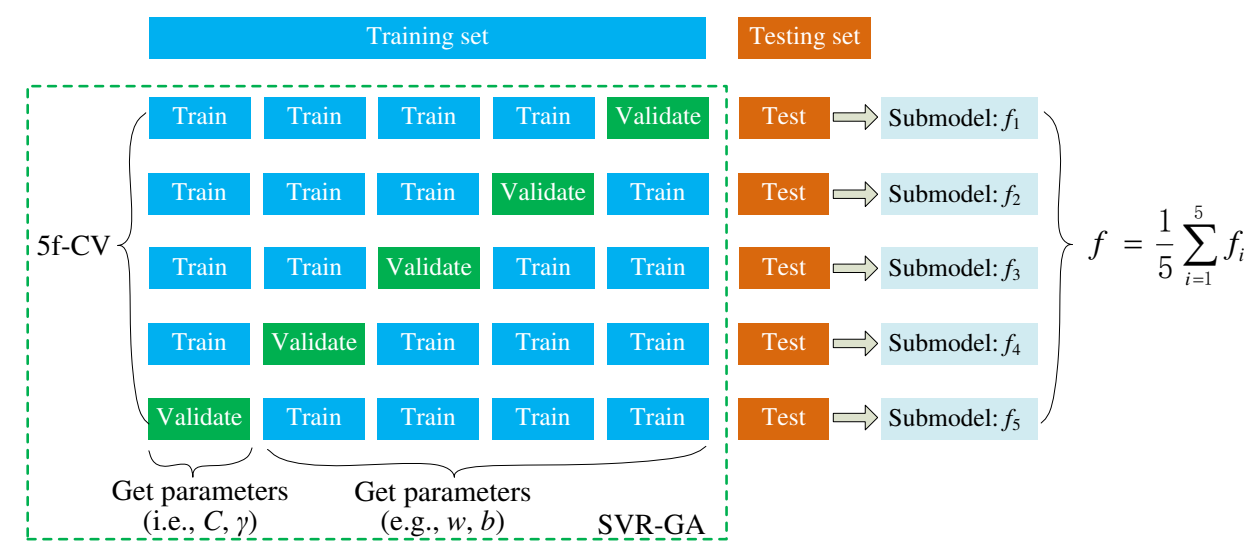

Fig. 3 Schematic diagram of the five-fold cross-validation and mean prediction model 
The experimental dataset was normalized with Eq. (5) before model training and testing. where $x_{\mathrm{n}}$ is the normalized value of experimental data $\left(x_{\text {real }}\right)$, between -1 and 1. $x_{\max }$ and $x_{\min }$ are the maximum and minimum values of $x_{\text {real }}$, respectively. After model predictions, the inverse normalization is needed for the predicted data shown in Eq. (6).

$$
\begin{gathered}
x_{\mathrm{n}}=2 \frac{x_{\mathrm{real}}-x_{\min }}{x_{\max }-x_{\min }}-1 \\
x_{\text {real }}=\frac{\left(x_{\mathrm{n}}+1\right)\left(x_{\max }-x_{\min }\right)}{2}+x_{\min }
\end{gathered}
$$

Here, five statistical metrics are used to measure performance of proposed model, such as coefficient of determination $\left(R^{2}\right)$, mean absolute error (MAE), root mean squared error (RMSE), mean square error (MSE). The closer the value of $R^{2}$ gets to 1 , the better the prediction achieves. The formula of $R^{2}$ can be shown as below.

$$
R^{2}=\frac{\left[\sum_{i=1}^{n}\left(y_{i}-\bar{y}\right)\left(\hat{y}_{i}+\overline{\hat{y}}\right)\right]^{2}}{\sum_{i=1}^{n}\left(y_{i}-\bar{y}\right)^{2} \sum_{i=1}^{n}\left(y_{i}-\bar{y}\right)^{2}}
$$

where $y_{i}$ and $\hat{y}_{i}$ are the $i$ th observed and predicted values, respectively. $\bar{y}$ and $\overline{\hat{y}}$ are mean values of observed and predicted, respectively. $n$ is the total number of observations. The low values of MAE and RMSE indicate good prediction accuracy of the model. MAE, MSE and RMSE are given by Eqs. (8)- (10).

$$
\begin{aligned}
\text { MAE } & =\sum_{i=1}^{n}\left|y_{i}-\hat{y}_{i}\right| / n \\
\text { MSE } & =\sum_{i=1}^{n}\left(y_{i}-\hat{y}_{i}\right)^{2} / n \\
\text { RMSE } & =\sqrt{\sum_{i=1}^{n}\left(y_{i}-\hat{y}_{i}\right)^{2} / n}
\end{aligned}
$$

\section{Results and discussion}

\subsection{Performances of SVR-GA and SVR model}

Performances of the SVR-GA model and SVR model are investigated and discussed based on the training set and testing set. SVR-GA and SVM models were both applied to learn the relationship between the normalized ultimate shear strength $\left(v_{\mathrm{u}}\right)$ and eight different input variables $\left(s, b, h_{0}, f_{\mathrm{c}}, f_{\mathrm{t}}, \rho\right.$ and $\left.\rho_{\mathrm{sv}} f_{\mathrm{yv}}\right)$. In order to visualize the results of the models, the experimental and predicted shear strength values versus the experiment number of training set are presented graphically in scatter diagrams shown in Fig. 4 and Fig. 5 for the SVR-GA model and SVR model, respectively. Plumb lines are drawn to indicate prediction errors between the values of experimental shear strength and predicted shear strength. It can be seen that most of the predicted points are closer to the experimental points with SVR-GA model compared to SVR model. It also reveals that the predicted errors in the SVR model are higher than SVR-GA model. Meanwhile, the predicted errors mainly appear in the region of high shear strength in both models, and they also increase with the increase of shear strength. One possible reason is that there are fewer experimental data points available in this region. 


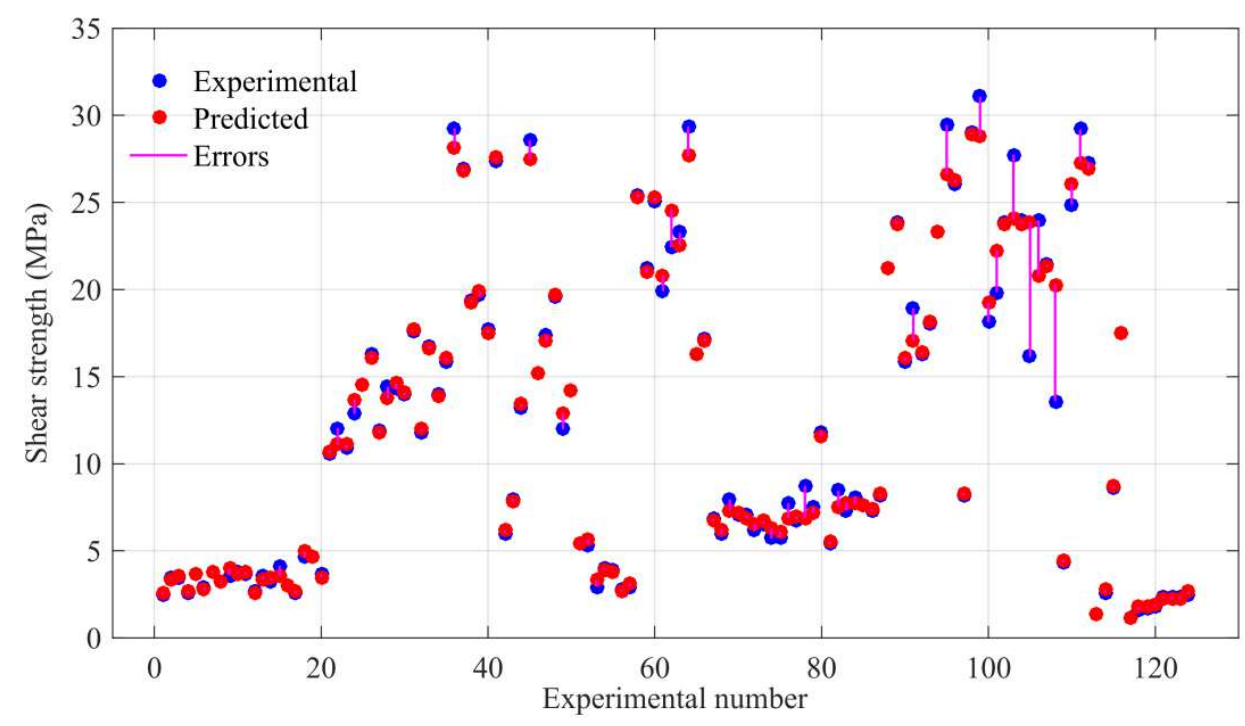

Fig. 4 The comparison of experimental strength to predicted strength for SVR-GA with training set

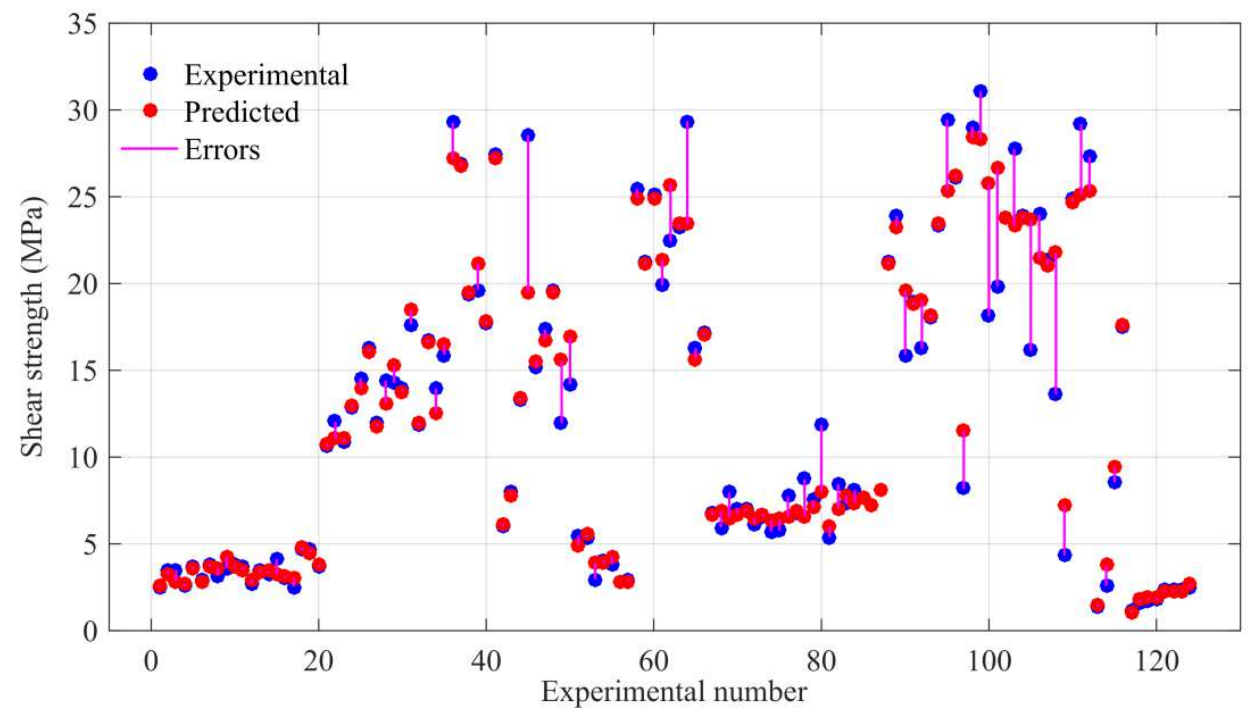

Fig. 5 The comparison of experimental strength to predicted strength for SVR with training set

The prediction error analysis box diagram of the ratio of experimental value to predicted value is shown graphically in Fig. 6. As a statistical diagram, the box diagram can provide key information about the location and dispersion of the data, and it can also clearly show the maximum, minimum, median, and upper and lower quartiles of the data. When the ratio of the predicted value to the test value is used as the input data of the box diagram, the closer the data point is to 1 and the lower the dispersion degree gets, the higher the accuracy the model can achieve. As seen in Fig. 6, the upper and lower quartiles and the median of the two models are close to 1 and the degree of dispersion is very small, especially for the SVR-GA model. It suggests that both SVR model and SVR-GA model can well predict the shear strength, and the SVR-GA model performs better than the SVR model. 


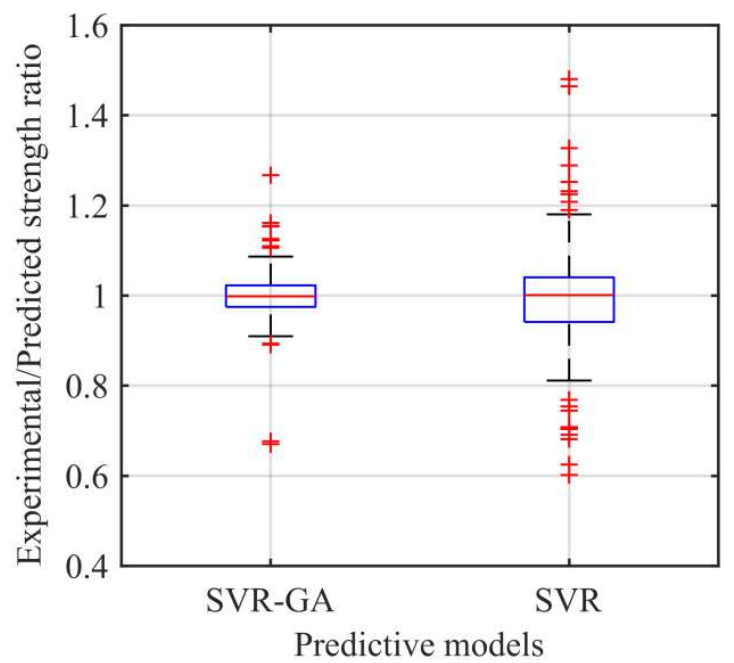

Fig. 6 Comparison of the model error of SVR-GA and SVR using the training data

The comparison of the experimental shear strength with the predicted value of the SVR-GA model and the SVR model are presented graphically in scatter plot shown in Figs. 7 and 8 for the training set and testing set, respectively. The fitting lines of the experimental and predicted value are also shown in the figures. It can be seen that the closer the fitting line is to the perfect line (the included angle with the $\mathrm{x}$-axis is to $45^{\circ}$ ), the better predicted performance the model works. The angle slope of the fitting line is close to the perfect line in both the training set and testing set. This validates the consistency of the models of SVR-GA and SVR. We can also derive that the predicted values of the SVR-GA model have less dispersion compared with the SVR model based on both training set and the testing set indicating that the SVR-GA model surpasses the SVR model.

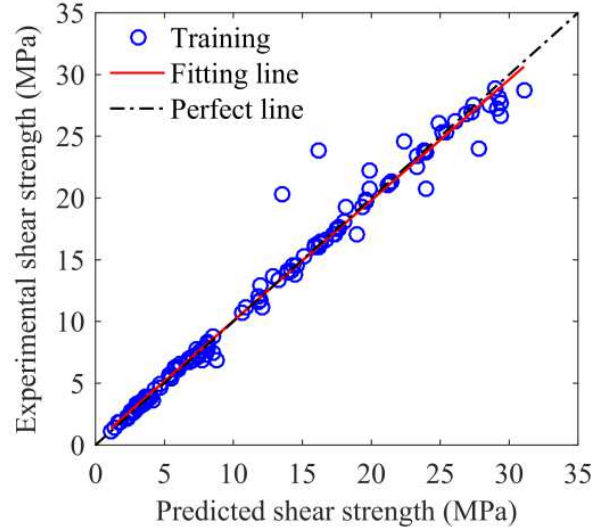

(a) SVR-GA model

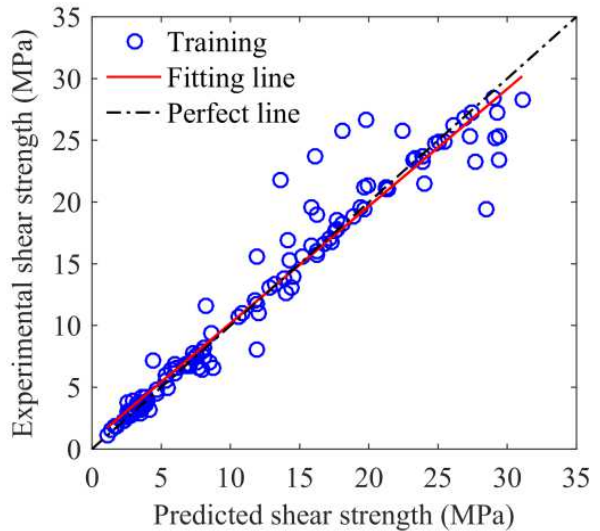

(b) SVR model

Fig. 7 The comparison of experimental strength to predicted strength using the training data 


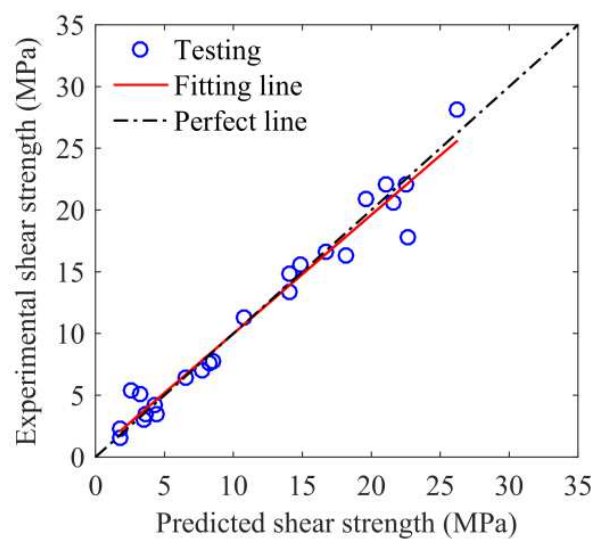

(a) SVR-GA model

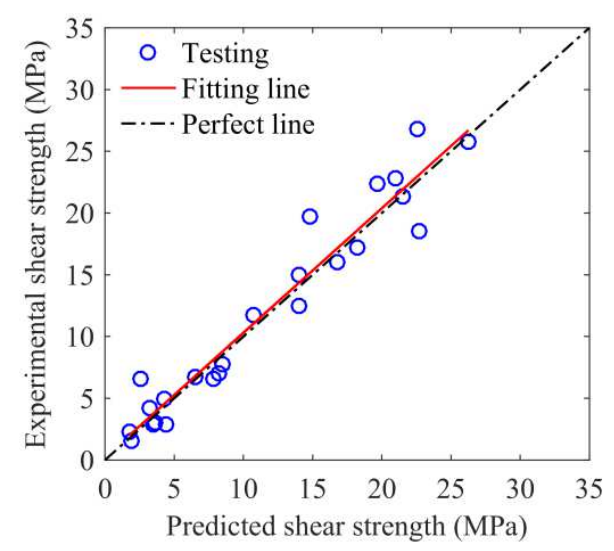

(b) SVR model

Fig. 8 The comparison of experimental strength to predicted strength using the testing data

To further determine the accuracies of the two models mentioned above, different metrics such as $R^{2}$, RMSE, MSE and MAE are measured by Eqs. (7)- (10) and the reported statistical results are shown in Table 2 for both the training set and testing set. The SVR-GA model performs better both in the training stage and testing stage recorded with higher values of $R^{2}$ and the lower values of RMSE, MSE and MAE. To be specific, for the training set, $R^{2}=0.9806$, RMSE $=$ $1.2055, \mathrm{MSE}=1.4533$ and $\mathrm{MAE}=0.5281$. Contrast to SVR model with $R^{2}=0.9419, \mathrm{RMSE}=$ 2.0882, MSE $=4.3605$ and MAE $=1.0773$. The SVR model performs a little worse than SVR-GA model based on the testing set with lower $R^{2}$ (0.9379), higher RMSE (2.0375), MSE (4.1513) and MAE (1.4973). While there were higher $R^{2}$ (0.9642), lower RMSE (1.4685), MSE (2.1566) and MAE (1.0216) for SVR-GA model. Fig. 9 reveals that the MAE and RMSE values of the SVR-GA model are lower than the SVR model. These results show that the SVR-GA model can better predict the ultimate shear strength. More details can be seen in Table 2 .

\begin{tabular}{lllll} 
Table 2 Statistical properties of the developed models. \\
\hline Parameters & \multicolumn{2}{l}{ SVR-GA model } & \multicolumn{2}{l}{ SVR model } \\
Dataset & Training & Testing & Training & Testing \\
& data & data & data & data \\
\hline$R^{2}$ & 0.9806 & 0.9642 & 0.9419 & 0.9379 \\
RMSE & 1.2055 & 1.4685 & 2.0882 & 2.0375 \\
MSE & 1.4533 & 2.1566 & 4.3605 & 4.1513 \\
MAE & 0.5281 & 1.0216 & 1.0773 & 1.4973 \\
Mean & 0.9988 & 0.9999 & 0.9949 & 0.9995 \\
Std & 0.0696 & 0.1745 & 0.1354 & 0.2181
\end{tabular}

\footnotetext{
${ }^{*}$ Mean and Std are respectively the mean and standard deviation of the ratio of the experimental shear strength to
} the predicted value. 


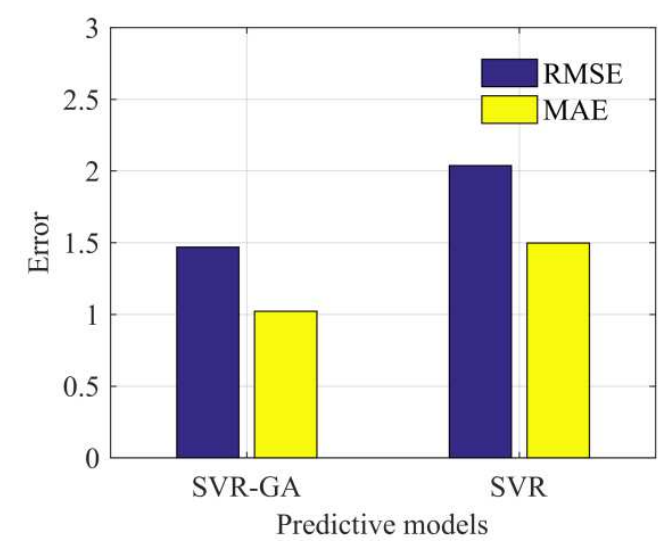

Fig. 9 Comparison of the RMSE and MAE of SVR-GA model and SVR model using the testing data

\subsection{Sensitivity Analysis}

The sensitivity analysis is implemented for exploring the important degree of each of the input variables using the SVR-GA model. Twentieth quantiles (from minimum to maximum, increased by 5\%) of each input variable collated from the experimental dataset are used as a new dataset to calculate the normalized ultimate shear strength. To be more precise, the value of one input varies from minimum to maximum while all other inputs remain with their average values. According to the statistical probability distribution of input variables, the influence of input variable changes on output results is explored shown in Table 3. For example, the minimum value (0.79) and maximum value (5.0) of $a / d$, and the median value of other parameters are taken to predict the shear strength as $v_{\max }(15.7606 \mathrm{MPa})$ and $v_{\min }(4.9361 \mathrm{MPa})$, then the important degree of this variable is $\left(v_{\max }-v_{\min }\right) / v_{\max }=0.6868$. The sensitivity analysis results for each input are presented graphically in bar chart shown in Fig. 10. The figure reveals that all input variables can affect the prediction of the shear strength through the SVR-GA model. The most important variables are $a / d, f_{\mathrm{c}}, \rho$ and $\rho_{\mathrm{sv}} f_{\mathrm{yv}}$, with the degree of importance values of $0.6868,0.6044,0.4615$ and 0.4607 , respectively. This information is highly relevant to the literature (Wang et al. 2020), and is consistent with the results of the literature that the most important parameters are the shear span-to-depth ratio and concrete compressive strength. However, Fig. 10 reveals that the stirrup spacing has little effect on the shear strength of the slender beam, and its important degree is only 0.1658. It is noteworthy that although the range of stirrup spacing in the experimental dataset is very large ranging from $20 \mathrm{~mm}$ to $1000 \mathrm{~mm}$, the median value is only $151 \mathrm{~mm}$, and most of the data are small. Consequently, a larger database of larger stirrup spacing should be considered in future research to explore the degree of importance of the stirrup spacing. 


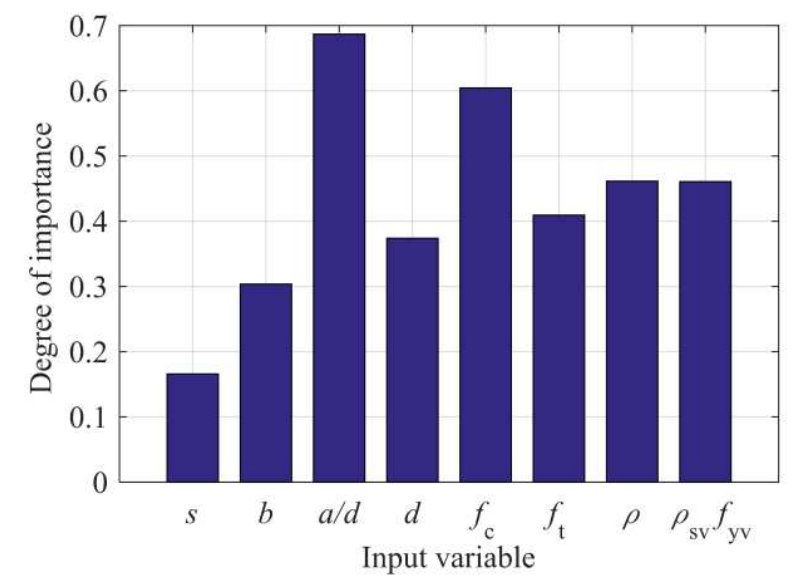

Fig. 10 Bar chart of the degree of importance value estimation

Table 3 Values of the variables used to calculate the degree of importance.

\begin{tabular}{cccccccccc}
\hline$s(\mathrm{~mm})$ & $b(\mathrm{~mm})$ & $a / d$ & $d(\mathrm{~mm})$ & $f_{\mathrm{c}}(\mathrm{MPa})$ & $f_{\mathrm{t}}(\mathrm{MPa})$ & $\rho(\%)$ & $\rho_{\text {sv }} f_{\mathrm{yv}}(\mathrm{MPa})$ & $v_{\mathrm{u}}(\mathrm{MPa})$ & DI $^{*}$ \\
\hline $\mathbf{1 0 0 0}$ & 122.47 & 2.67 & 277.4 & 109.6 & 7.49 & 3.78 & 274.29 & 15.1803 & \\
$\mathbf{6 0}$ & 122.47 & 2.67 & 277.4 & 109.6 & 7.49 & 3.78 & 274.29 & 12.6631 & 0.1658 \\
179.1 & $\mathbf{2 0 0}$ & 2.67 & 277.4 & 109.6 & 7.49 & 3.78 & 274.29 & 11.5489 & \\
179.1 & $\mathbf{4 0}$ & 2.67 & 277.4 & 109.6 & 7.49 & 3.78 & 274.29 & 16.5844 & 0.3036 \\
179.1 & 122.47 & $\mathbf{5 . 0 0}$ & 277.4 & 109.6 & 7.49 & 3.78 & 274.29 & $\mathbf{4 . 9 3 6 1}$ & \\
179.1 & 122.47 & $\mathbf{0 . 7 9}$ & 277.4 & 109.6 & 7.49 & 3.78 & 274.29 & $\mathbf{1 5 . 7 6 0 6}$ & $\mathbf{0 . 6 8 6 8}$ \\
179.1 & 122.47 & 2.67 & $\mathbf{4 3 0}$ & 109.6 & 7.49 & 3.78 & 274.29 & 7.1733 & \\
179.1 & 122.47 & 2.67 & $\mathbf{1 1 4}$ & 109.6 & 7.49 & 3.78 & 274.29 & 11.4559 & 0.3738 \\
179.1 & 122.47 & 2.67 & 277.4 & $\mathbf{2 3 2 . 1}$ & 7.49 & 3.78 & 274.29 & 23.7992 & \\
179.1 & 122.47 & 2.67 & 277.4 & $\mathbf{2 3 . 6}$ & 7.49 & 3.78 & 274.29 & 9.4155 & 0.6044 \\
179.1 & 122.47 & 2.67 & 277.4 & 109.6 & $\mathbf{2 4 . 1 8}$ & 3.78 & 274.29 & 16.2389 & \\
179.1 & 122.47 & 2.67 & 277.4 & 109.6 & $\mathbf{1 . 9 6}$ & 3.78 & 274.29 & 9.5910 & 0.4094 \\
179.1 & 122.47 & 2.67 & 277.4 & 109.6 & 7.49 & $\mathbf{1 3 . 5 7}$ & 274.29 & 18.3481 & \\
179.1 & 122.47 & 2.67 & 277.4 & 109.6 & 7.49 & $\mathbf{0 . 5}$ & 274.29 & 9.8808 & 0.4615 \\
179.1 & 122.47 & 2.67 & 277.4 & 109.6 & 7.49 & 3.78 & $\mathbf{1 0 4 5}$ & 20.0627 & \\
179.1 & 122.47 & 2.67 & 277.4 & 109.6 & 7.49 & 3.78 & $\mathbf{1 2 . 0 2}$ & 10.8193 & 0.4607 \\
\hline
\end{tabular}

*DI denotes the degree of importance

The sensitivity analysis described above shows that machine learning and artificial intelligence technologies can be helpful during the design of beam shear resistance phase. In addition to accurately predicting the ultimate shear strength, the SVR-GA model can also help create more informative input/output maps of the ultimate shear strength. Especially, the most important variables ( $a / d, f_{\mathrm{c}}, \rho$ and $\left.\rho_{\mathrm{sv}} f_{\mathrm{yv}}\right)$ will be used here to illustrate the input and output maps of the ultimate shear strength. The values of the other variables remain the same as the average value. Six ultimate shear strength maps with the same color range are presented in Fig. 11, which shows the relationship diagrams of $f_{\mathrm{c}}$ and $a / d, f_{\mathrm{c}}$ and $\rho_{\mathrm{sv}} f_{\mathrm{yv}}, f_{\mathrm{c}}$ and $\rho, a / d$ and $\rho_{\mathrm{sv}} f_{\mathrm{yv}}, a / d$ and $\rho, \rho$ and $\rho_{\mathrm{sv}} f_{\mathrm{yv}}$, respectively. Three-dimensional maps indicate that there is a nonlinear behavior in the input and output relationship, which makes it difficult to detect their relationship only from the experimental data. Except that the increase of ald will reduce the shear strength to some extent, the increase of 
other input variables will increase the shear strength to varying degrees. Figs. 11a and 11f show an interesting phenomenon that the ultimate shear strength is not the maximum, when the two most important factors ( $a / d$ and $f_{\mathrm{c}}$ ) are both used as inputs. On the contrary, the ultimate shear strength reaches the maximum, when taking the two lower important factors ( $\rho$ and $\left.\rho_{\mathrm{sv}} f_{\mathrm{yv}}\right)$ as inputs. This reflects from the side that the shear strength calculation of the beam with stirrups is extremely complex, which is the result of the coupling of multiple variables, and the variables cannot be considered separately.

(a)

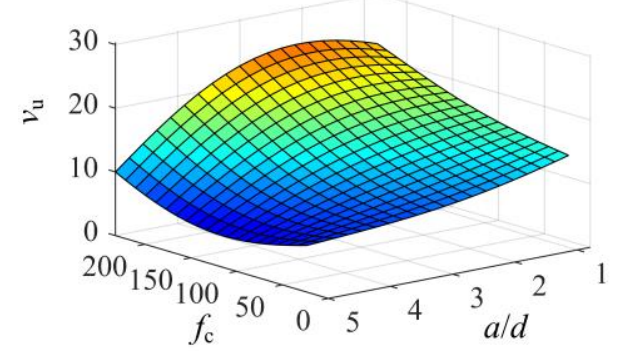

(c)

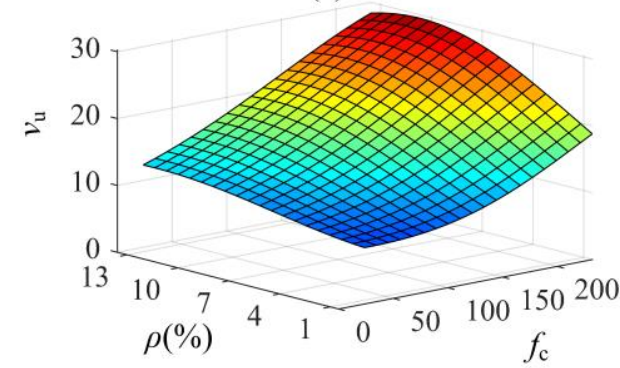

(e)

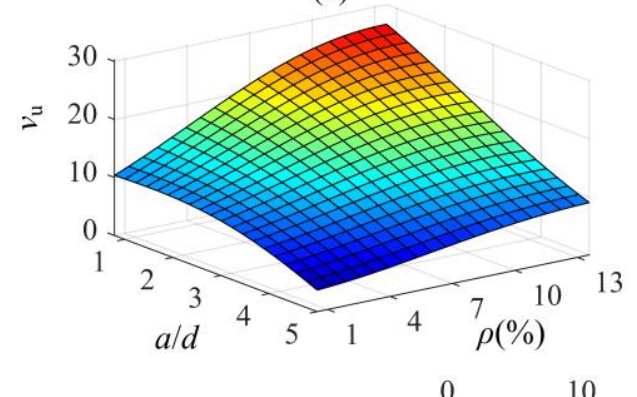

(b)

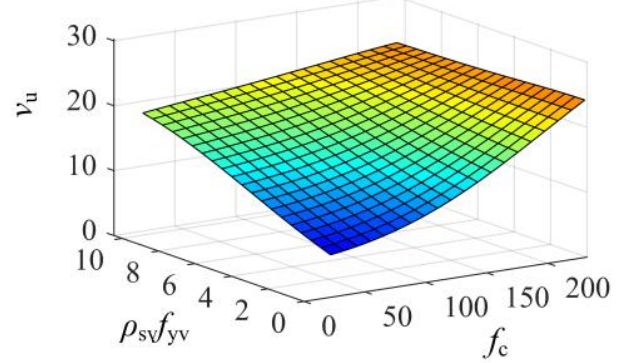

(d)

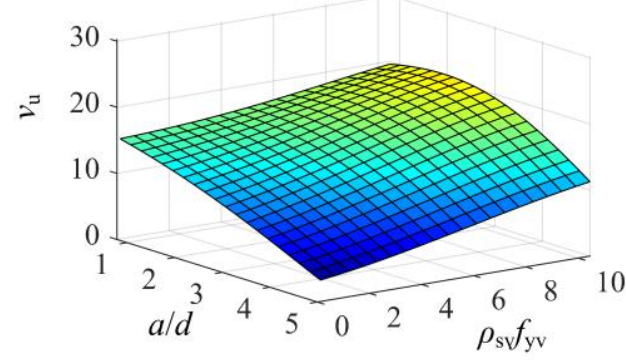

(f)

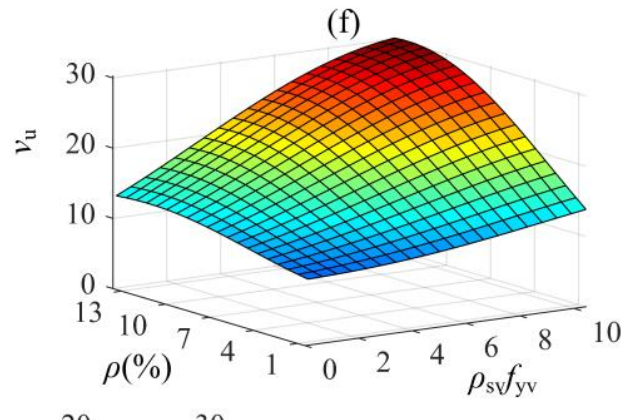

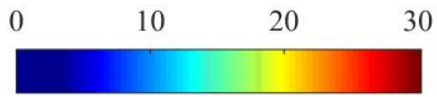

Fig. 11 Three-dimensional input and output maps of the ultimate shear strength: (a) $f_{\mathrm{c}}-a / d$, (b) $f_{\mathrm{c}}-\rho_{\mathrm{sv}} f_{\mathrm{yv}}$, (c) $f_{\mathrm{c}}-\rho$, (d) $a / d-\rho_{\mathrm{sv}} f_{\mathrm{yv}}$, (e) $a / d-\rho$, and (f) $\rho-\rho_{\mathrm{sv}} f_{\mathrm{yv}}$.

\section{Conclusions}

Due to the complexity of the shear design of ultra-high concrete strength beams with stirrups, current research investigates the feasibility of using machine learning techniques of the SVR-GA and SVR model to predict the shear strength of medium- to ultra-high strength concrete beams with stirrups. In order to improve the accuracy of the SVR model, biological heuristic algorithm (GA) is introduced to determine the optimal SVR parameters more efficiently to obtain the perfect 
predictive model. To improve the model generalization ability, a five-fold cross-validation approach was employed to train the model preventing from over-fitting. Experiment results show that SVR-GA model has better performance than the traditional SVR model, and its $R^{2}$ value in the training set is 0.9806 , and the $R^{2}$ value in the testing set is 0.9642 . The SVR-GA model in this study can make the more accurate prediction for shear strength. The sensitivity analysis indicates that all input variables can affect the prediction accuracy of the shear strength, and the most important variables are $a / d, f_{\mathrm{c}}, \rho$ and $\rho_{\mathrm{sv}} f_{\mathrm{yv}}$. The model proposed here can guide the shear design. At the same time, there are still some issues that need to be resolved. For example, we only studied the shear strength of medium-to ultra-high strength concrete beams with stirrups, and ignored the other properties. These will be the focuses of our research in the future.

Funding: The authors are very grateful for the support of the Guangxi Basic Ability Promotion Project for Young and Middle-aged Teachers (No. 2018KY0241). At the same time, we would also like to thank the distinguished reviewers and editors for their excellent suggestions and comments.

Conflict of interest: The authors declare no conflict of interest.

Human and animal rights: This article does not include any studies of human participants performed by the authors.

\section{CRediT authorship contribution statement}

Chun-Song Jiang: Conceptualization, Methodology, Formal analysis, Writing \& editing.

Gui-Qin Liang: Supervision, Resources, Review \& editing.

\section{References}

ACI Committee 318 (2014) Aci 318-14

Açikgenç M, Ulaş M, Alyamaç KE (2015) Using an Artificial Neural Network to Predict Mix Compositions of Steel Fiber-Reinforced Concrete. Arab J Sci Eng 40:. https://doi.org/10.1007/s13369-014-1549-x

Baby F, Marchand P, Toutlemonde F (2014) Shear Behavior of Ultrahigh Performance Fiber-Reinforced Concrete Beams. I: Experimental Investigation. J Struct Eng 140: https://doi.org/10.1061/(asce)st.1943-541x.0000907

Ccoicca YJ (2013) Applications of Support Vector Machines in the Exploratory Phase of Petroleum and Natural Gas: a Survey. Int J Eng Technol 2:. https://doi.org/10.14419/ijet.v2i2.834

Çevik A, KURTOĞLU AE, Bilgehan M, et al (2015) Support vector machines in structural engineering: A review. J. Civ. Eng. Manag. 21

Chatterjee S, Sarkar S, Hore S, et al (2017) Structural failure classification for reinforced concrete buildings using trained neural network based multi-objective genetic algorithm. Struct Eng Mech 63:. https://doi.org/10.12989/sem.2017.63.4.429

Collins MP, Bentz EC, Sherwood EG, Wight JK (2009) Where is shear reinforcement required? review of research results and design procedures. ACI Struct. J. 106

Farfani HA, Behnamfar F, Fathollahi A (2015) Dynamic analysis of soil-structure interaction using the neural networks and the support vector machines. Expert Syst Appl 42: 
https://doi.org/10.1016/j.eswa.2015.07.053

Golafshani EM, Rahai A, Sebt MH (2015) Artificial neural network and genetic programming for predicting the bond strength of GFRP bars in concrete. Mater Struct Constr 48:.

https://doi.org/10.1617/s11527-014-0256-0

Guo M, Hesthaven JS (2018) Reduced order modeling for nonlinear structural analysis using Gaussian process regression. Comput Methods Appl Mech Eng 341:. https://doi.org/10.1016/j.cma.2018.07.017

Hasgul U, Yavas A, Birol T, Turker K (2019) Steel fiber use as shear reinforcement on I-shaped UHP-FRC beams. Appl Sci 9:. https://doi.org/10.3390/app9245526

Hassan AMT, Jones SW, Mahmud GH (2012) Experimental test methods to determine the uniaxial tensile and compressive behaviour of Ultra High Performance Fibre Reinforced Concrete(UHPFRC). Constr Build Mater 37:. https://doi.org/10.1016/j.conbuildmat.2012.04.030

Hoang ND, Pham AD, Nguyen QL, Pham QN (2016) Estimating Compressive Strength of High Performance Concrete with Gaussian Process Regression Model. Adv Civ Eng 2016:. https://doi.org/10.1155/2016/2861380

Hossain KMA, Gladson LR, Anwar MS (2017) Modeling shear strength of medium- to ultra-high-strength steel fiber-reinforced concrete beams using artificial neural network. Neural Comput Appl 28: https://doi.org/10.1007/s00521-016-2417-2

Hou LJ, Luan ZY, Chen D, Xu SL (2015) Experimental study of the shear properties of reinforced ultra-high toughness cementitious composite beams. J Zhejiang Univ Sci A 16: https://doi.org/10.1631/jzus.A1400274

Ji W, Ding B, An M (2011) Experimental study on the shear capacity of reactive powder concrete T-beams. Zhongguo Tiedao Kexue/China Railw Sci 32:

Jin LZ, Li YX, Qi KN, He P (2015) Research on shear bearing capacity and ductility of high strength reinforced RPC beam. Gongcheng Lixue/Engineering Mech 32: https://doi.org/10.6052/j.issn.1000-4750.2014.04.S056

Kamal MM, Safan MA, Etman ZA, Salama RA (2014) Behavior and strength of beams cast with ultra high strength concrete containing different types of fibers. HBRC J 10:. https://doi.org/10.1016/j.hbrcj.2013.09.008

Krassowska J, Kosior-Kazberuk M, Berkowski P (2019) Shear behavior of two-span fiber reinforced concrete beams. Arch Civ Mech Eng 19:. https://doi.org/10.1016/j.acme.2019.09.005

Lim WY, Hong SG (2016) Shear Tests for Ultra-High Performance Fiber Reinforced Concrete (UHPFRC) Beams with Shear Reinforcement. Int J Concr Struct Mater 10:. https://doi.org/10.1007/s40069-016-0145-8

Magureanu C, Sosa I, Negrutiu C, Heghes B (2010) Bending and shear behavior of ultra-high performance fiber reinforced concrete. In: WIT Transactions on the Built Environment

Mansouri I, Ozbakkaloglu T, Kisi O, Xie T (2016) Predicting behavior of FRP-confined concrete using neuro fuzzy, neural network, multivariate adaptive regression splines and M5 model tree techniques. Mater Struct Constr 49:. https://doi.org/10.1617/s11527-015-0790-4

Marì Bernat A, Spinella N, Recupero A, Cladera A (2020) Mechanical model for the shear strength of steel fiber reinforced concrete (SFRC) beams without stirrups. Mater Struct Constr 53: https://doi.org/10.1617/s11527-020-01461-4

Mészöly T, Randl N (2018) Shear behavior of fiber-reinforced ultra-high performance concrete beams. Eng Struct 168:. https://doi.org/10.1016/j.engstruct.2018.04.075

Ministry of Transport of China (2018) Specifications for Design of Highway Reinforced Concrete and Prestressed Concrete Bridges and Culverts JTG 3362-2018. People's Communications Press, Beijing 
Nguyen QH, Ly HB, Le TT, et al (2020) Parametric investigation of particle swarm optimization to improve the performance of the adaptive neuro-fuzzy inference system in determining the buckling capacity of circular opening steel beams. Materials (Basel) 13:. https://doi.org/10.3390/ma13102210

Olalusi OB, Viljoen C (2020) Model uncertainties and bias in SHEAR strength predictions of slender stirrup reinforced concrete beams. Struct Concr 21:316-332. https://doi.org/10.1002/suco.201800273

Pal M, Deswal S (2011) Support vector regression based shear strength modelling of deep beams. Comput Struct 89:. https://doi.org/10.1016/j.compstruc.2011.03.005

Pansuk W, Nguyen TN, Sato Y, et al (2017) Shear capacity of high performance fiber reinforced concrete I-beams. Constr Build Mater 157:. https://doi.org/10.1016/j.conbuildmat.2017.09.057

Press CSI (2010) Code for design of concrete structures GB 50010-2010. China Struct. Sci. Acad. Beijing

Qi JN, Wang JQ, Zhou K, et al (2020) Experimental and Theoretical Investigations on Shear Strength of UHPC Beams. Zhongguo Gonglu Xuebao/China J Highw Transp 33:. https://doi.org/10.19721/j.cnki.1001-7372.2020.07.010

Russo G, Somma G, Angeli P (2004) Design shear strength formula for high strength concrete beams. Mater Struct Constr 37:. https://doi.org/10.1617/14016

Sagaseta J, Vollum RL (2011) Influence of beam cross-section, loading arrangement and aggregate type on shear strength. Mag Concr Res 63:. https://doi.org/10.1680/macr.9.00192

Słowik M (2014) Shear Failure Mechanism in Concrete Beams. Procedia Mater Sci 3:. https://doi.org/10.1016/j.mspro.2014.06.318

Smarzewski P (2018) Hybrid fibres as shear reinforcement in high-performance concrete beams with and without openings. Appl Sci 8:. https://doi.org/10.3390/app8112070

Solhmirzaei R, Salehi H, Kodur V, Naser MZ (2020) Machine learning framework for predicting failure mode and shear capacity of ultra high performance concrete beams. Eng Struct 224:. https://doi.org/10.1016/j.engstruct.2020.111221

Taylor CE (1994) Adaptation in Natural and Artificial Systems: An Introductory Analysis with Applications to Biology, Control, and Artificial Intelligence. Complex Adaptive Systems. John H. Holland . Q Rev Biol 69:. https://doi.org/10.1086/418447

Umeonyiagu IE, Nwobi-Okoye CC (2019) Modelling and multi objective optimization of bamboo reinforced concrete beams using ANN and genetic algorithms. Eur J Wood Wood Prod 77:. https://doi.org/10.1007/s00107-019-01418-7

Vapnik V, Golowich SE, Smola A (1997) Support vector method for function approximation, regression estimation, and signal processing. In: Advances in Neural Information Processing Systems

Wang Q, Song HL, Lu CL, Jin LZ (2020) Shear performance of reinforced ultra-high performance concrete rectangular section beams. Structures 27:. https://doi.org/10.1016/j.istruc.2020.07.036

Xu H, Deng Z, Chen C, Chen X (2014) Experimental study on shear strength of ultra-high performance fiber reinforced concrete beams. Tumu Gongcheng Xuebao/China Civ Eng J 47:

Yan F, Lin Z (2016) New strategy for anchorage reliability assessment of GFRP bars to concrete using hybrid artificial neural network with genetic algorithm. Compos Part B Eng 92:. https://doi.org/10.1016/j.compositesb.2016.02.008

Yoo DY, Yoon YS (2016) A Review on Structural Behavior, Design, and Application of Ultra-High-Performance Fiber-Reinforced Concrete. Int J Concr Struct Mater 10:. https://doi.org/10.1007/s40069-016-0143-X

Yousef AM, Tahwia AM, Marami NA (2018) Minimum shear reinforcement for ultra-high performance fiber reinforced concrete deep beams. Constr Build Mater 184: https://doi.org/10.1016/j.conbuildmat.2018.06.022 
Yu PS, Chen ST, Chang IF (2006) Support vector regression for real-time flood stage forecasting. J Hydrol 328:. https://doi.org/10.1016/j.jhydrol.2006.01.021

Zhang G, Ali ZH, Aldlemy MS, et al (2020) Reinforced concrete deep beam shear strength capacity modelling using an integrative bio-inspired algorithm with an artificial intelligence model. Eng Comput. https://doi.org/10.1007/s00366-020-01137-1

ZHANG HZ (2005) Experimental Study on Shear Performance of High Strength Concrete Beams. Dalian University of Technology, Dalian

Zheng H, Fang Z, Chen B (2019) Experimental study on shear behavior of prestressed reactive powder concrete I-girders. Front Struct Civ Eng 13:. https://doi.org/10.1007/s11709-018-0500-8

Zhou JM, Chen S (2015) Experimental study and Evaluation of the mechanical properties of reinforced concrete structures with High strength. Science Press, Beijing 


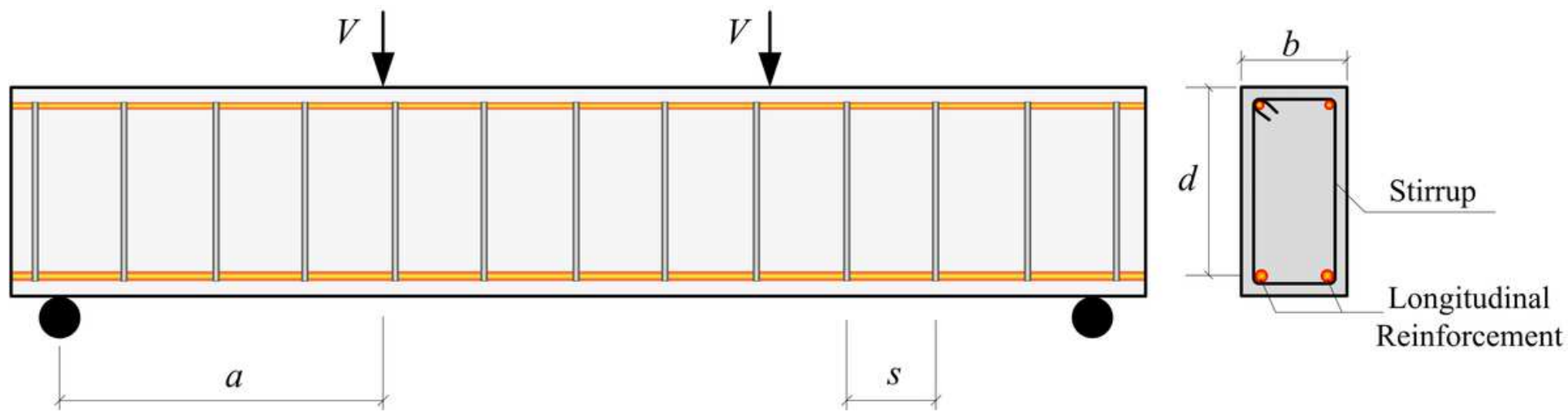

Figure 1

Typical RC beam with stirrups and its geometric parameters

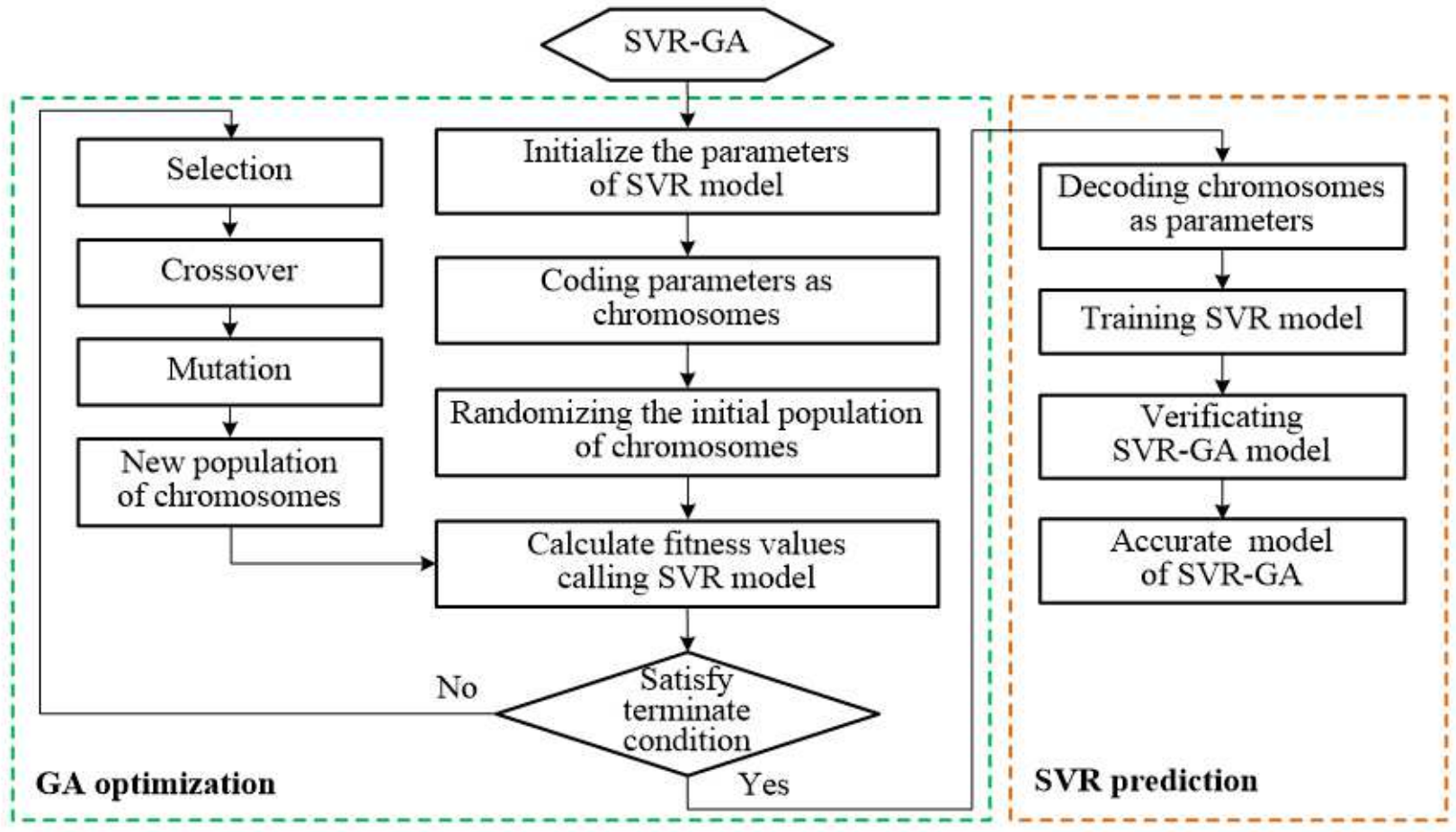

Figure 2

Flow diagram of hybrid SVR-GA model 


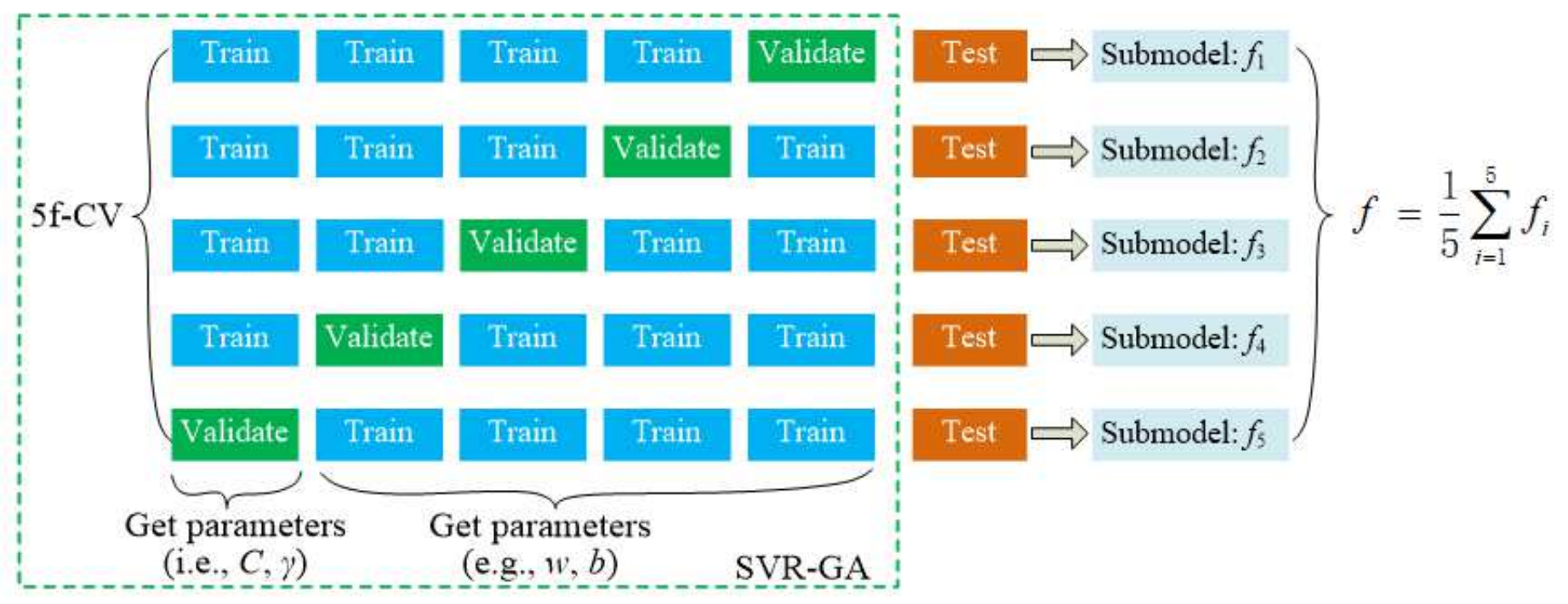

Figure 3

Schematic diagram of the five-fold cross-validation and mean prediction model

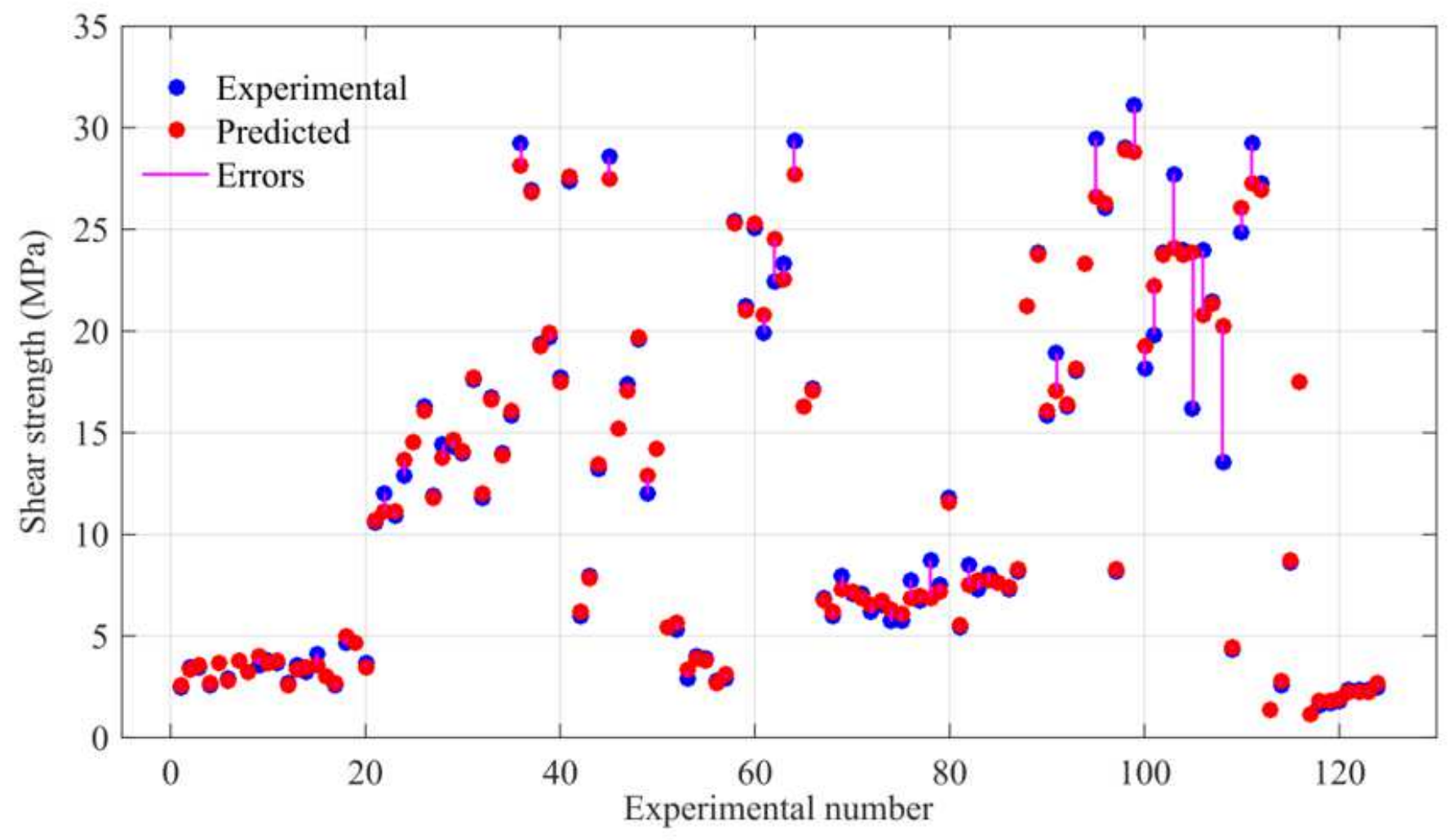

Figure 4

The comparison of experimental strength to predicted strength for SVR-GA with training set 


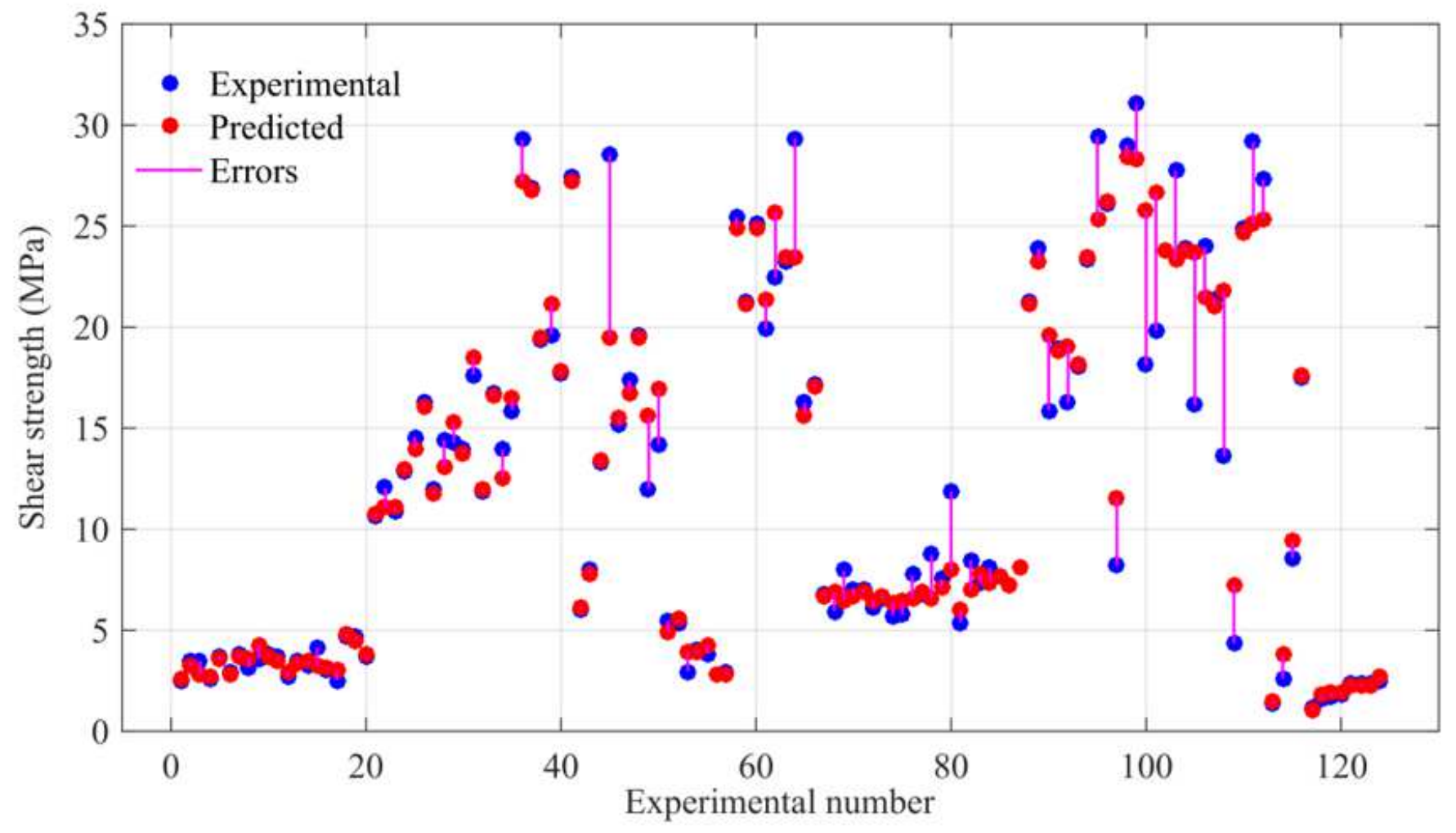

Figure 5

The comparison of experimental strength to predicted strength for SVR with training set 


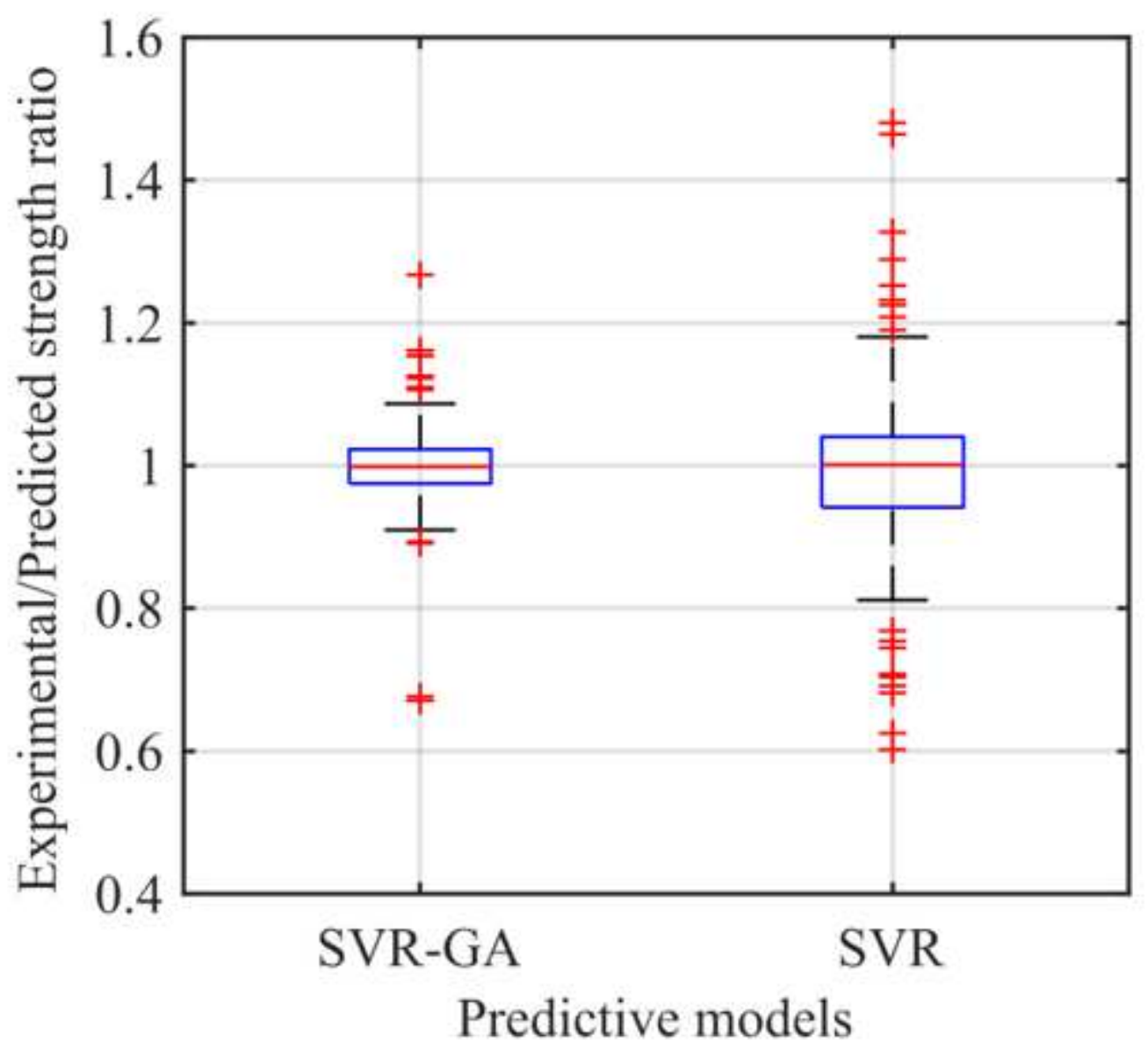

Figure 6

Comparison of the model error of SVR-GA and SVR using the training data

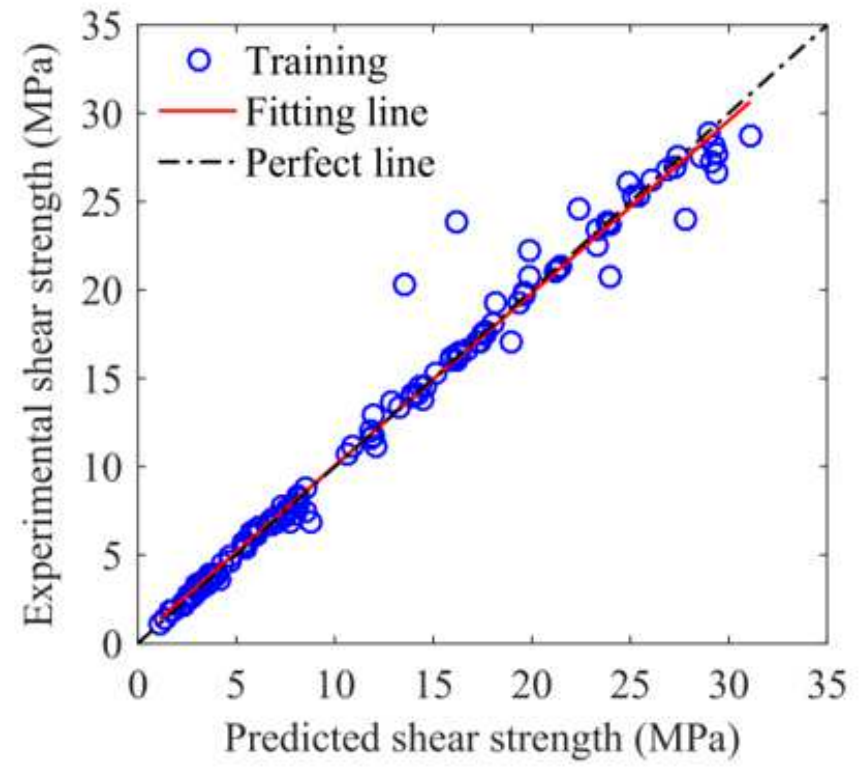

(a) SVR-GA model

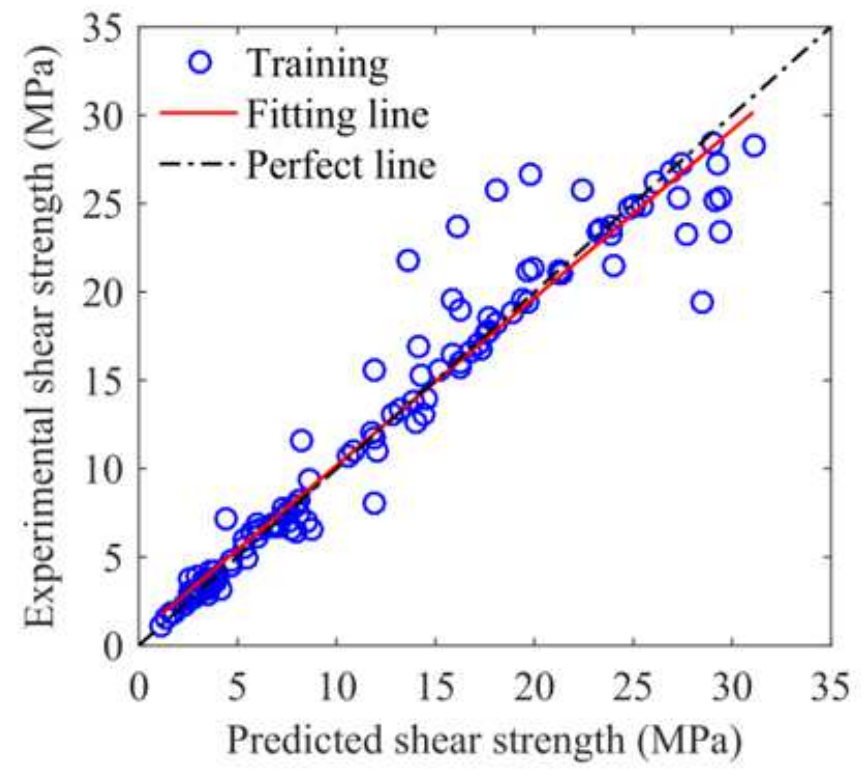

(b) SVR model 
Figure 7

The comparison of experimental strength to predicted strength using the training data

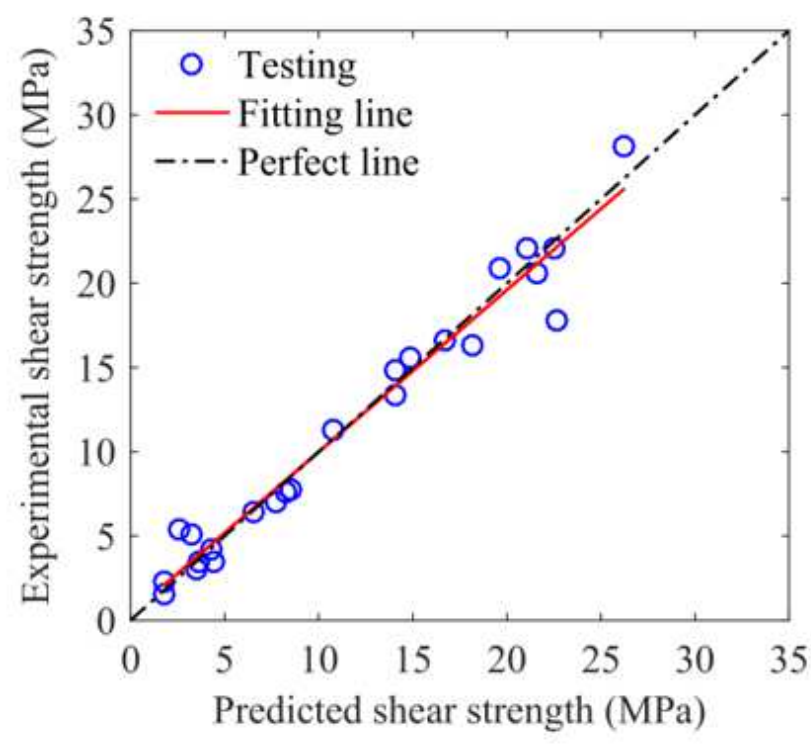

(a) SVR-GA model

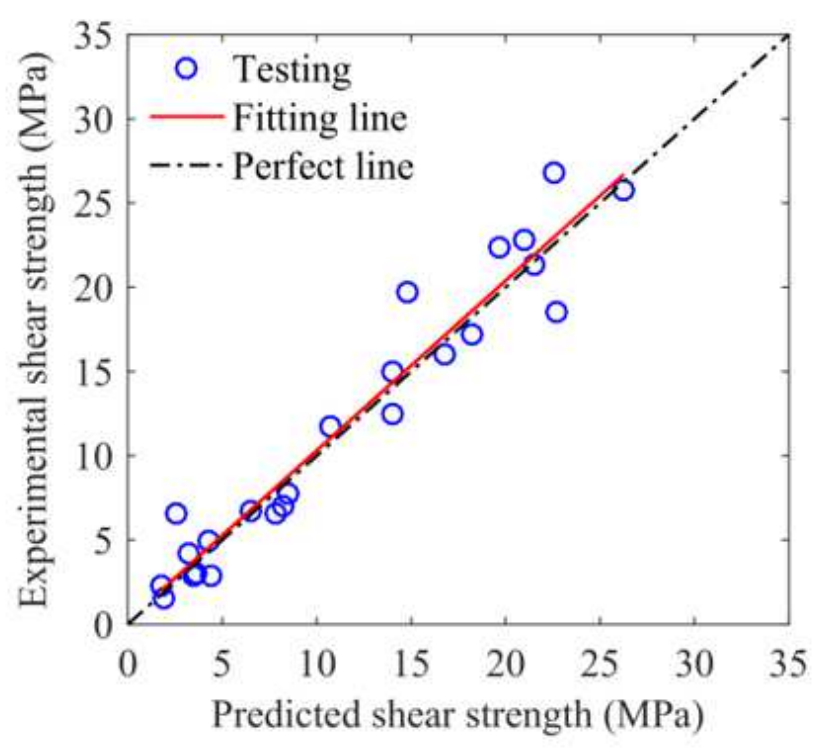

(b) SVR model

Figure 8

The comparison of experimental strength to predicted strength using the testing data

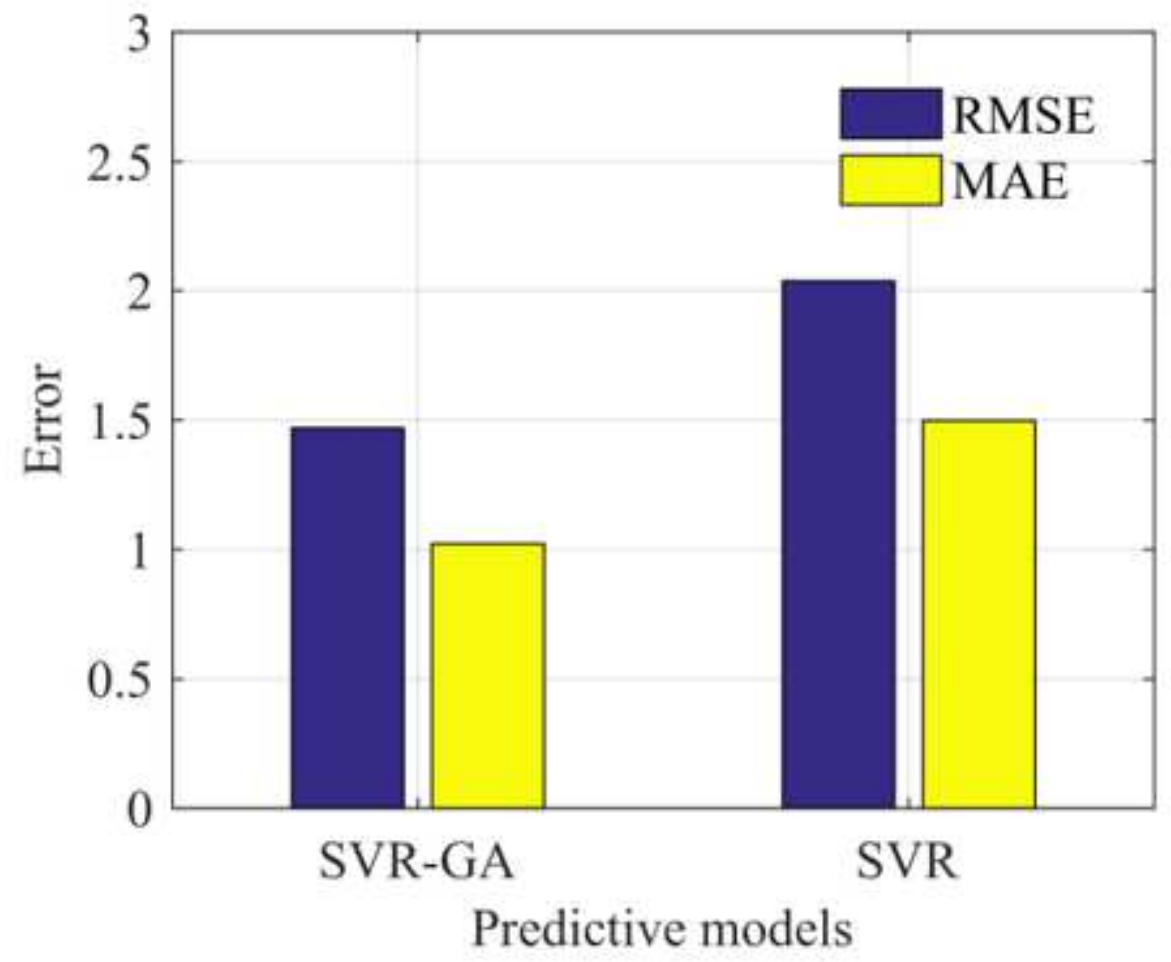

Figure 9 
Comparison of the RMSE and MAE of SVR-GA model and SVR model using the testing data

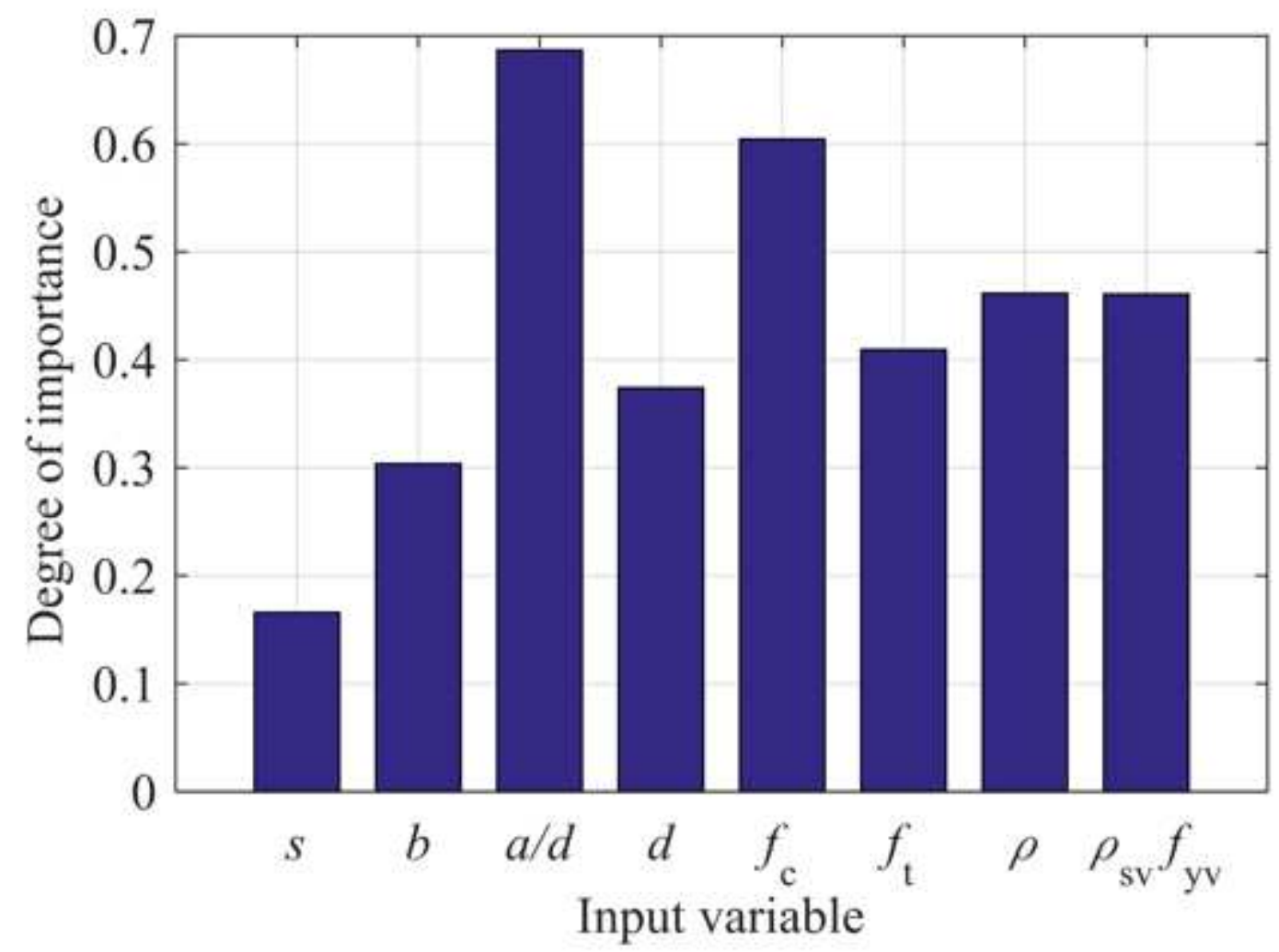

Figure 10

Bar chart of the degree of importance value estimation 
(a)

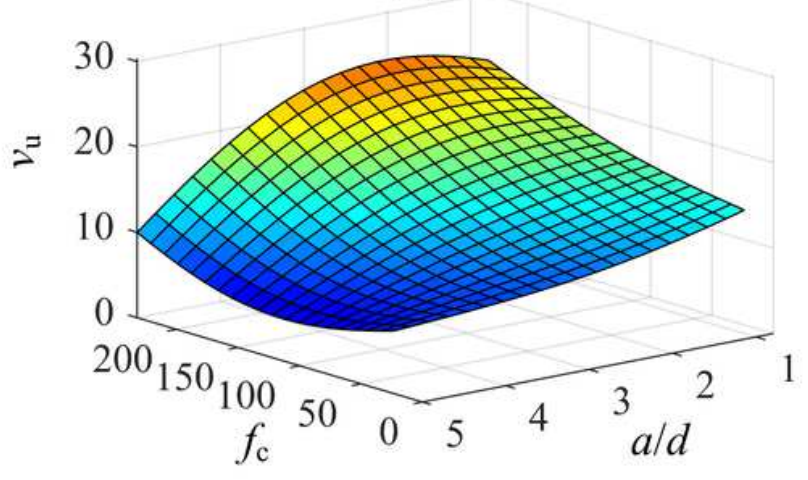

(c)

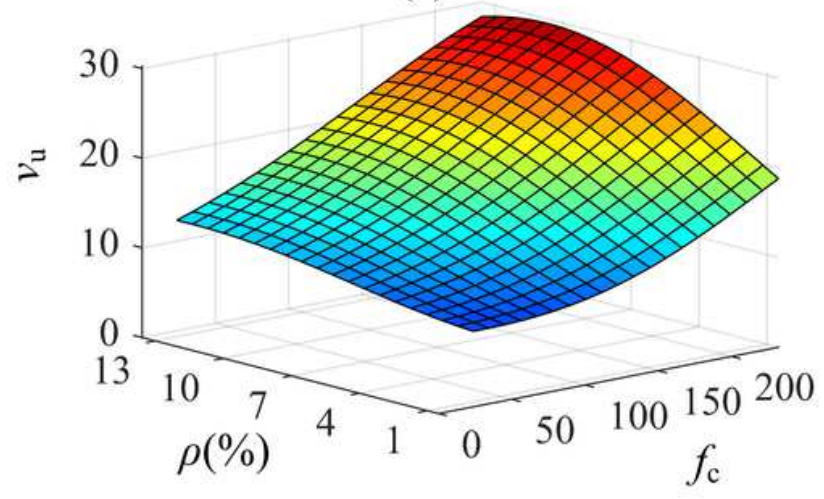

(e)

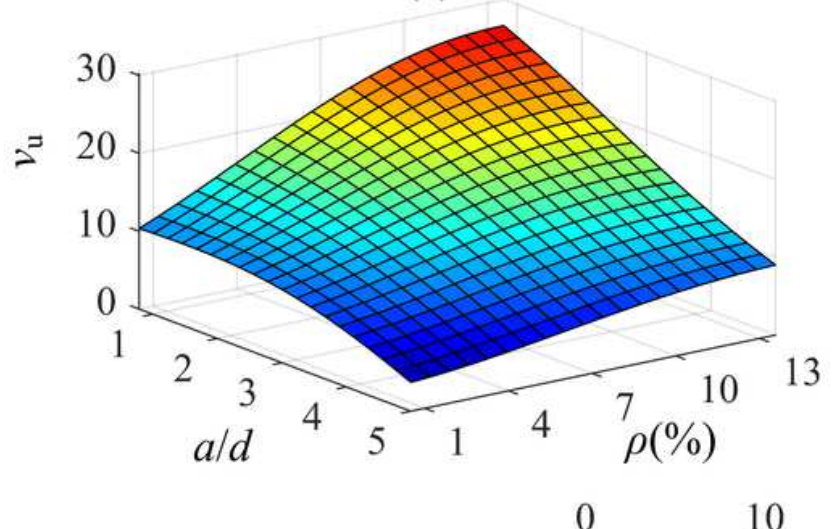

(b)

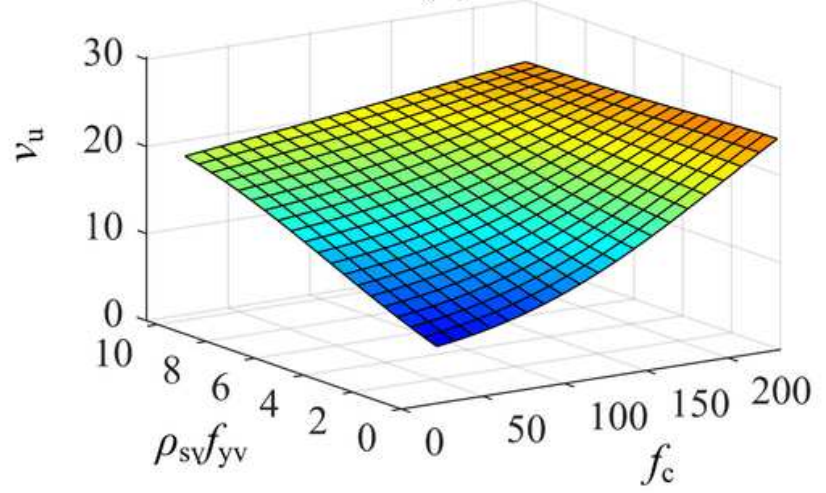

(d)

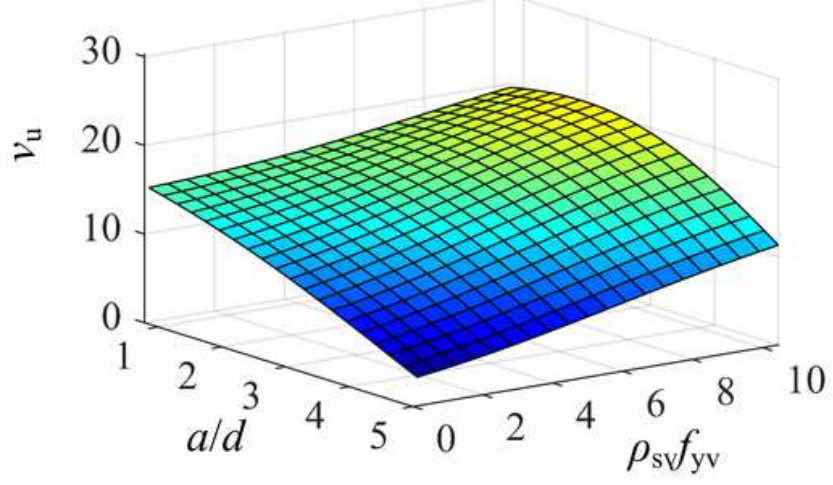

(f)

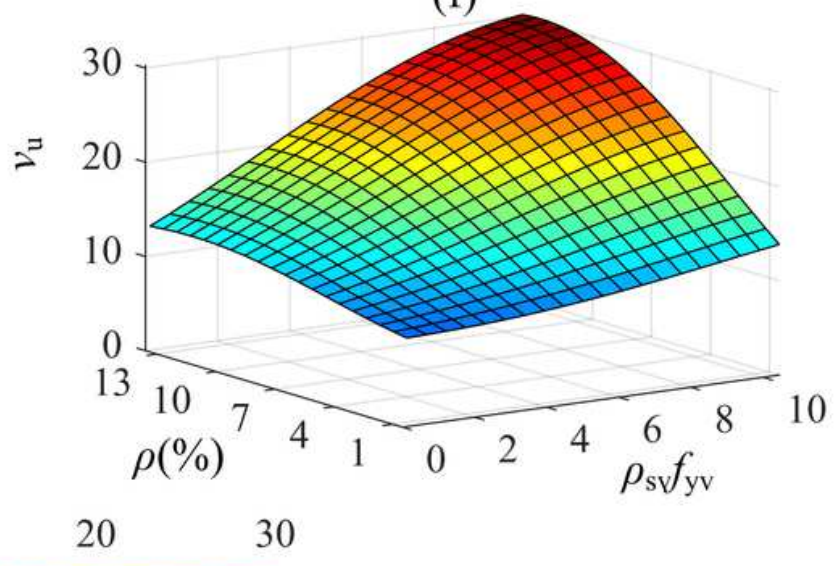

\section{Figure 11}

Three-dimensional input and output maps of the ultimate shear strength: (a) fc-a/d, (b) fc-psvfyv, (c) fc-p, (d) a/d-psvfyv, (e) a/d-p, and (f) $\rho$-psvfyv. 